
NATIONAL INSTITUTE OF STANDAROG \&

TECHNOLOGY

Research Information Center

Gaithersourg, MD 20898 



\section{NBSIR 88-3830}

\section{The ICST-NBS Information Resource Dictionary System Command Language Prototype}

Alan Goldfine

Thomasin Kirkendall

U.S. DEPARTMENT OF COMMERCE

National Bureau of Standards

Institute for Computer Sciences and Technology

Gaithersburg, MD 20899

August 1988
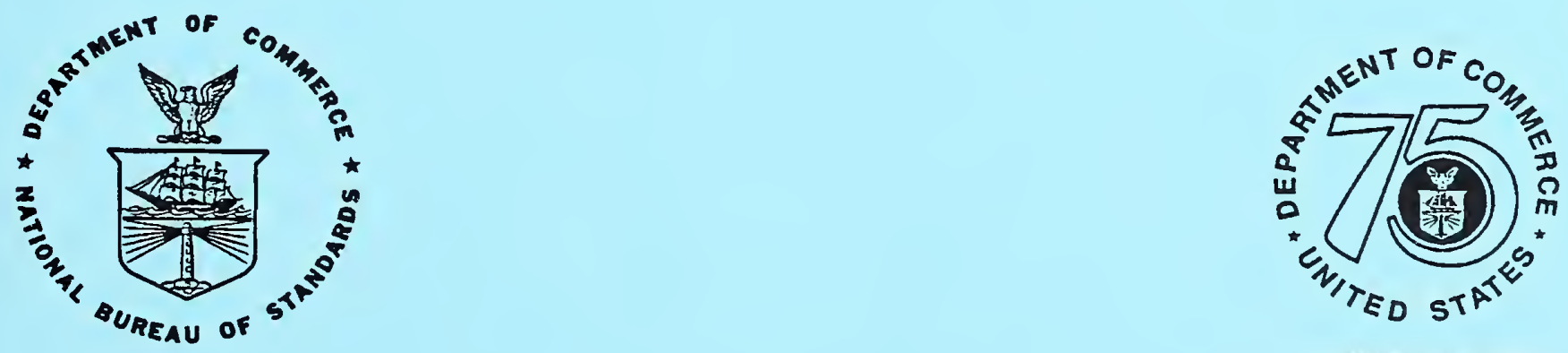

75 Yeres Shimulating Americe's Progreses

1813-1989

U.S. DEPARTMENT OF COMMERCE

NATIONAL BUREAU OF STANDARDS 



\section{THE ICST-NBS INFORMATION RESOURCE}

DICTIONARY SYSTEM COMMAND LANGUAGE PROTOTYPE

Alan Goldfine

Thomasin Kirkendall

U.S. DEPARTMENT OF COMMERCE

National Bureau of Standards

Institute for Computer Sciences and Technology

Gaithersburg, MD 20899

August 1988

U.S. DEPARTMENT OF COMMERCE, C. William Verity, Secretary NATIONAL BUREAU OF STANDARDS, Ernest Ambler, Director 



\title{
THE ICST-NBS INFORMATION RESOURCE DICTIONARY SYSTEM COMMAND LANGUAGE PROTOTYPE
}

\author{
Alan Goldfine \\ Thomasin Kirkendall
}

\begin{abstract}
This publication is a report on the Information Resource Dictionary system (IRDS) Command Language prototype developed by the Institute for computer sciences and Technology of the National Bureau of standards. It discusses the structure, source code, and operating environment of the IRDS Prototype, specifies the precise subset of the standard IRDS Command Language implemented, provides instructions for installing the prototype software, and leads the reader through a typical user session.

Key words: command language; data dictionary; data dictionary system; Information Resource Dictionary System; IRDS; prototype.
\end{abstract}

\section{ACKNOWLEDGMENTS}

We would like to gratefully acknowledge the summer trainees and coop students, working at ICST-NBS, who contributed to the programming, installation, testing, and documentation of the Prototype: Sam Cook, Joe Farrington, Jim Gould, Richard Morris, and Doug White. 
Page iv 
TABLE OF CONTENTS

Page

1. AN OVERVIEW OF THE IRDS PROTOTYPE . . . . . . . . . . 1

1.1 HISTORY . . . . . . . . . . . . . . . . . . . . . 1

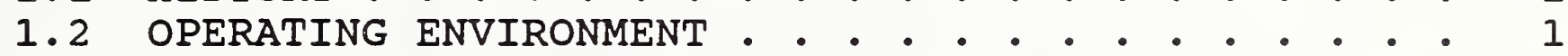

1.3 DISTRIBUTION OF THE IRDS PROTOTYPE . . . . . . . 2

1.4 SCOPE AND USE OF THIS REPORT . • • • • • . . . . . 3

2. AN IRDS PROTOTYPE SESSION . • • . . . . . . . . . . . . 4

3. THE IRDS PROTOTYPE COMMAND IANGUAGE • • • • • • . . 6

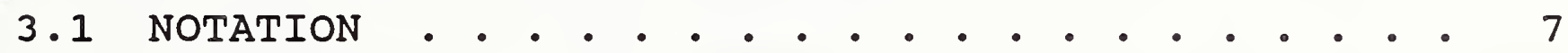

3.2 IRD COMMANDS . . . . . . . . . . . . . . . . 47

3.2.1 ADD ENTITY . . . . . . . . . . . . . . . . 7

3.2 .2 MODIFY ENTITY • . . . . . . . . . . . . 8

3.2 .3 DELETE ENTITY • . . . . . . . . . . . . . . 9

3.2 .4 ADD RELATIONSHIP • • • • • • • • . . . . . . . 9

3.2 .5 MODIFY RELATIONSHIP . . . . . . . . . . . . 10

3.2.6 DELETE RELATIONSHIP . • . . . • . . . . . . . 11

3.2 .7 MODIFY ENTITY ACCESS-NAME . . . . . . . . . . 11

3.2.8 MODIFY ENTITY DESCRIPTIVE-NAME • • . . . • . . 12

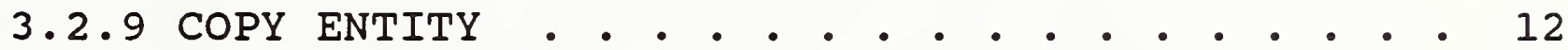

3.2.10 GENERAL OUTPUT . . . . . . . . . . . . . 13

3.3 IRD-SCHEMA COMMANDS . . . . . . . . . . . . . 15

3.3.1 ADD META-ENTITY . . . . . . . . . . . . . 15

3.3.2 MODIFY META-ENTITY . . . . . . . . . . . 16

3.3.3 DELETE META-ENTITY • • • . . • . . • • . . 16

3.3.4 ADD META-RELATIONSHIP . . . . . . . . . . . . 17

3.3 .5 MODIFY META-RELATIONSHIP . . . . . . . . . . . 17

3.3.6 DELETE META-RELATIONSHIP . . . . . . . . . . . 18

3.3.7 MODIFY META-ENTITY ACCESS-NAME . . . . . . . 18

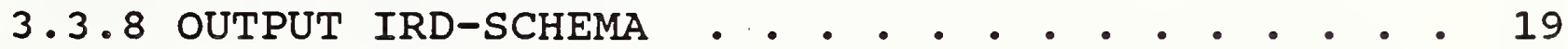

3.4 UTILITY COMMANDS . . . . . . . . . . . . . . . . 21

3.4.1 CREATE IRD . . . . . . . . . . . . . . . . . . 21

3.4.2 REMOVE IRD . . . . . . . . . . . . . . . . . . 22

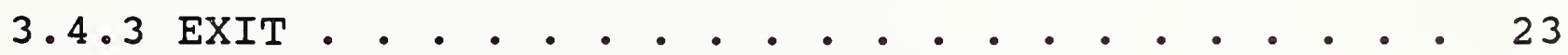

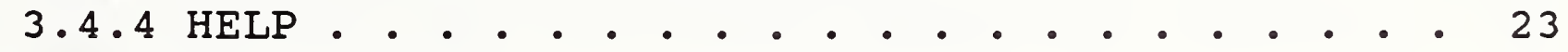

3.5 ERROR MESSAGES . . . . . . . . . . . . . . . 23

3. 6 COMMAND LANGUAGE ABBREVIATIONS . . . . . . . . . 24

4. THE IRDS PROTOTYPE SCHEMA . . . . . . . . . . . 25

4.1 THE STRUCTURE OF THE SQL TABLES . . . . . . • . . 25

4.1.1 The META-ATTRIBUTE-TYPE Table . . . . . . . . 25

4.1.2 The META-ATTRIBUTE-GROUP-TYPE/META-ATTRIBUTE-

TYPE Table............. . . 26 
Page

4.1.3 The META-ENTITY-TYPE/META-ATTRIBUTE-TYPE Table 26

4.1.4 The META-ENTITY-TYPE/META-ATTRIBUTE-GROUP-TYPE Table . . . . . . . . . . . . . 28

4.1.5 The META-ENTITY/META-ATTRIBUTE Table . . . . 28

4.1.6 The META-ENTITY/META-ATTRIBUTE-GROUP Table . . 30

4.1.7 The META-RELATIONSHIP-TYPE/META-ATTRIBUTE-TYPE Table . . . . . . . . . . . . . 30

4.1.8 The META-RELATIONSHIP/META-ATTRIBUTE Table . . 31

4.1.9 The ENTITY/ATTRIBUTE Table . . . . . . . . . 32

4.1.10 The ENTITY/ATTRIBUTE-GROUP Table . . . . . 33

4.1.11 The RELATIONSHIP/ATTRIBUTE Table • . • . . . 33

4.2 IMPLEMENTOR DEFINED VALUES IN THE IRDS PROTOTYPE 34

4.2.1 Values For Meta-Attribute-Types . . . . . . 34

4.2.2 Values For Meta-Entities . . . . . . . . . 36

5. THE IRDS PROTOTYPE SOURCE CODE - . . . . . . . . . 42

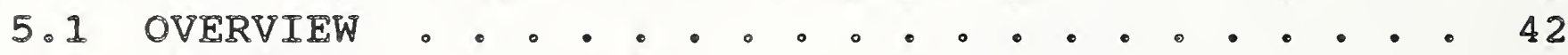

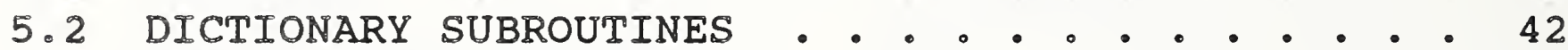

5.3 PARSING THE COMMANDS . . . . . . . . . . . . 43

5.4 COMMAND SUBROUTINES $. . \cdot . \cdot . \cdot . \cdot . \cdot . . .44$

5.5 OCI SUBROUTINES . . . . . . . . . . . . . . . 44

5.6 HLI SUBROUTINES . . . . . . . . . . . . . . . . . 44

5.7 GLOBAL VARIABLES • . . . . . . . . . . . . . 45

5.8 PROGRAM DATA STRUCTURES . . . . . . . . . . . . 45

6. INSTALLATION INSTRUCTIONS - . . . . . . . . . . . . 47 


\section{AN OVERVIEW OF THE IRDS PROTOTYPE}

\subsection{HISTORY}

Specifications for the Information Resource Dictionary system (IRDS), the emerging standard for data dictionary software, have been under development since 1980 as a joint effort of the Institute for Computer Sciences and Technology of the National Bureau of Standards (ICST-NBS) and Technical Committee $\mathrm{H} 4$ of the Accredited standards committee $\mathrm{X} 3$ (X3H4) [1].

Because the IRDS specifications, in particular those for the IRDS Command Language, describe a system quite different from currently available commercial data dictionary systems, ICST-NBS decided to develop a prototype Command Language implementation. The initial goal was to produce an IRDS prototype that would serve as a tool allowing experimentation on, and testing of, both the overall IRDS capabilities and the particular command Language syntax. Later, this IRDS prototype would be available for use by organizations wishing to become familiar with the upcoming standard.

The IRDS Prototype was developed and used for testing the Specifications during 1985-1986. This coincided with the period of public and Federal Government agency review of the IRDS. In 1986, ICST-NBS began distributing the IRDS Prototype source code to interested outside organizations. In 1988, ICST-NBS released a revised version of the IRDS Prototype that is compatible with the final, standard specifications.

\subsection{OPERATING ENVIRONMENT}

The Prototype uses SQI calls to the ORACLE' database management system to model the IRDS data structures and to provide the underlying data management. A set of $C$ language programs interpret the Prototype commands and interface with the DBMS.

ORACLE was chosen as the DBMS because it was available, was appropriate for the task, and because it implemented the

1 ORACLE is a registered trademark of Oracle corporation. 
SQL standard. This use, however, should not be considered an endorsement or certification of the ORACLE product.

The Prototype is designed to be independent of the particular hardware environment and operating system of the system hosting the C compiler and oracle DBMS.

\subsection{DISTRIBUTION OF THE IRDS PROTOTYPE}

The source code for the Prototype is available free of charge to interested organizations. The code is distributed on $51 / 4$ inch, double-sided, double-density diskettes, stored in ASCII text file format. The files are readable by any computer using the DOS operating system.

ICST-NBS is distributing the Prototype to allow organizations to experiment with the emerging IRDS standard Command Language. Users are encouraged to evaluate the Prototype software, and the underlying IRDS specifications, for correctness, design philosophy, and desirable enhancements. Users are also asked to provide ICST-NBS with feedback concerning their experiences with the Prototype.

Users of the IRDS Prototype must agree to fully identify and credit ICST-NBS as the developer of the Prototype in any publications, talks, reports, or products that are based on work utilizing the Prototype.

The ICST-NBS IRDS Prototype is in the public domain, and no restrictions are placed on its use. It is not subject to copyright in the United States. ICST-NBS provides no warranty, and is exempt of any liability.

To find out more about the IRDS Prototype, or to request a copy of the source code, please contact:

IRDS Prototype Project

National Bureau of Standards

Information Systems Engineering Division

Building 225, Room A266

Gaithersburg, MD 20899

Tel: (301) 975-3252 


\subsection{SCOPE AND USE OF THIS REPORT}

The remainder of this report begins with a detailed depiction of a typical IRDS Prototype session, including a discussion of how to create new dictionaries. Chapter 3 follows with a description of the Prototype command Language, including a description of the available commands, clauses, error messages, and allowable abbreviations. Chapter 4 discusses the structure of the SQL tables that store the IRD data and "implementor defined" parameter values used by the Prototype. In Chapter 5, the source code that implements the Prototype user interface is discussed. Much of the material in Chapters 4 and 5 may be of interest primarily to dictionary administrators. Finally, chapter 6 presents a detailed set of instructions for installing the Prototype software.

This report deals only with the ICST-NBS Prototype. It does not provide a complete description of the IRDS Standard, the details of the command Language, or any guidelines on IRDS usage. We recommend that users read the IRDS Technical Overview [2] as a tutorial and a general reference. A discussion, with many examples, of the complete Command Language is found in [3]. Guidelines for IRDS applications are presented in [4], and a guide on data entity naming conventions, within the framework of the IRDS, can be found in [5]. 


\section{AN IRDS PROTOTYPE SESSION}

Once the Prototype software has been installed, according to directions in Chapter 6, a user accesses the Prototype by running the executable file.

A session begins with the display of some package information giving the Prototype version number and the date that version was compiled. This is followed by the request:

IRDS_user_name :

The Prototype has no facilities for validating the user name that is entered: the information is used exclusively for audit purposes, such as for ADDED-BY attributes.

The Prototype then asks:

Is this a batch or interactive run (b/i)?

If the user enters "b", each user command is echoed, so the command string itself will be recorded as part of the batch output copy. An "i" specifies no echoing of the command string, and so is the normal response for a user working at a terminal.

Since each copy of the Prototype can support 25 discrete dictionaries, the prototype will, in general, display at this point a menu of all previously created IRDs:

Available IRDs are:

a) <name of first IRD>

b) <name of second IRD>

c) <name of third IRD>

Please specify your choice (letter) 
The user must select one of the specified choices, even if he or she intends to create a new IRD.

The Prototype acknowledges the selection with

The current IRD is <name of IRD>

The Prototype then places the user "in" the selected IRD, and returns the prompt symbol " $>$ ". If the selected IRD is the one desired, the user can now begin working. If, on the other hand, the user wishes to create a new IRD, he or she does so at this point, using CREATE IRD (see section 3.3.1).

If there are no previously created IRDs to select from, the Prototype will not display the above menu of existing IRDs, but will generate an implicit CREATE IRD command, and display the following:

INFORMATION IXXXX: Creating 1st schema table
INFORMATION IXXXX: Creating 2nd schema table
INFORMATION IXXXX: Creating 3rd schema table
INFORMATION IXXXX: Creating lst data table
INFORMATION IXXXX: Creating 2nd data table
INFORMATION IXXXX: Creating 3rd data table
INFORMATION IXXXX: All done.

The current IRD has no name.

What name do you want to give it?

The Prototype names the new IRD, displays

The current IRD is <name of IRD>

places the user in this IRD, and returns the prompt symbol ">".

It should be emphasized that the IRDS Command Language requires the use of the semicolon as the terminator of a command. The Prototype will take no action, and will remain in a wait state if the user forgets to place a semicolon at the end of a command. 


\title{
3. THE IRDS PROTOTYPE COMMAND LANGUAGE
}

The IRDS Prototype currently implements the following 21 commands :

\section{IRD Commands}

\author{
ADD ENTITY \\ MODIFY ENTITY \\ DELETE ENTITY \\ ADD RELATIONSHIP \\ MODIFY RELATIONSHIP \\ DELETE RELATIONSHIP \\ MODIFY ENTITY ACCESS-NAME \\ MODIFY ENTITY DESCRIPTIVE-NAME \\ COPY ENTITY \\ OUTPUT IRD
}

\section{IRD-Schema commands}

ADD META-ENTITY

MODIFY META-ENTITY

DELETE META-ENTITY

ADD META-RELATIONSHIP

MODIFY META-RELATIONSHIP

DELETE META-RELATIONSHIP

MODIFY META-ENTITY ACCESS-NAME

OUTPUT IRD-SCHEMA

\section{Utility Commands}

\author{
CREATE IRD \\ REMOVE IRD \\ EXIT \\ HELP
}

The HELP facility, in addition to providing users with on-line assistance, also serves to document the precise subset of the IRDS command Language implemented in the current version of the Prototype.

The following sections present, for each implemented command, the subset of the command Language syntax that has been included in the prototype, along with one or more examples of the command's use. The format of the Prototype's response to a correctly specified command is also described, as are any differences between the Prototype implementation 
and the standard Command Language, as defined in the IRDS Specifications [1], and discussed in [2] and [3].

\subsection{NOTATION}

The construct $\overbrace{B}^{A}$ in the syntax listings below

represents a choice between the clauses $A$ and $B$.

Words in capitals, such as ADD, ENTITY, and DESCRIPTIVENAME, are IRDS-defined words.

Angle brackets "<" and ">" enclose syntactic categories, e.g., "<access-name>" and "<attribute-clause>".

Square brackets "[" and "]" enclose optional items. A string of the form $[,\langle C\rangle$... ] represents the occurrence of zero or more instances of syntactic category $C$.

\subsection{IRD COMMANDS}

\section{2 .1 ADD ENTITY}

\section{Syntax:}

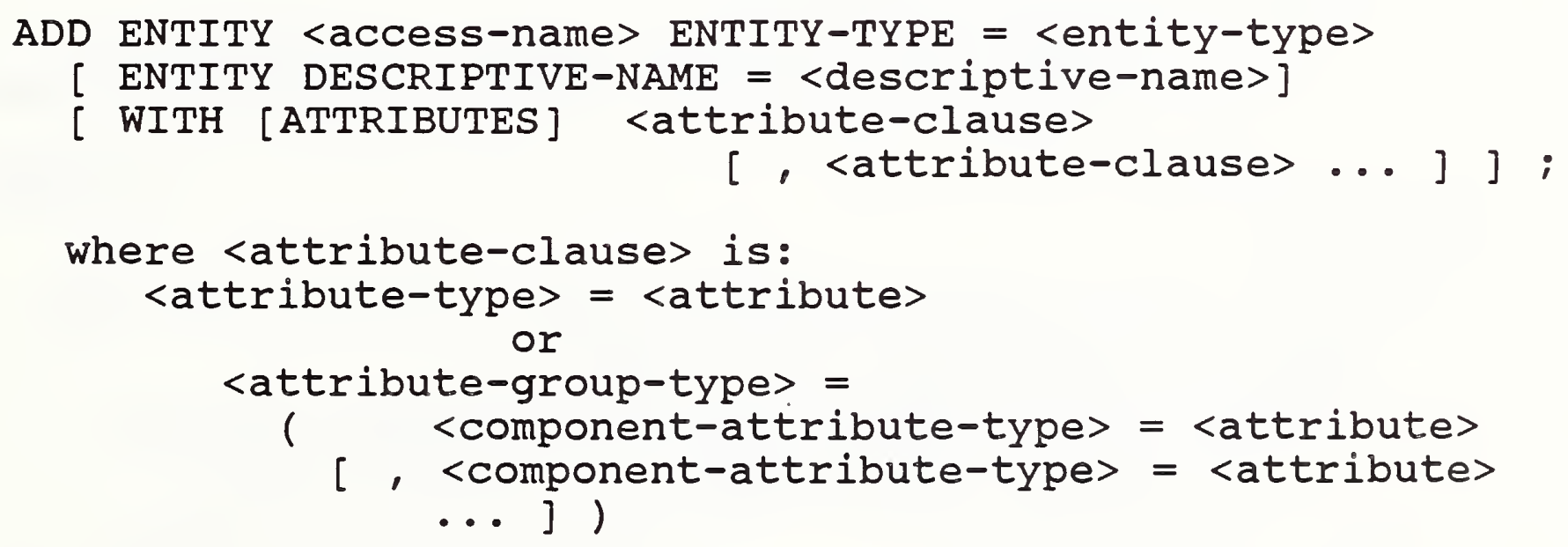

\section{Examples:}

add entity us entity-type = system;

add entity us entity-type = system

entity descriptive-name = example_system 
with comments = "this is an example system";

add entity u8 entity-type = system

with external-security = "none",

location = "example book",

identification-names =

(alternate-name = "example",

alternate-name-context $=$ "here");

Prototype Response:

Entity <access-name> added.

\subsubsection{MODIFY ENTITY}

\section{Syntax:}

MODIFY ENTITY <access-name>

[ ENTITY DESCRIPTIVE-NAME = <descriptive-name> ]

[ WITH [ ATTRIBUTES] <attribute-clause>

$[$, <attribute-clause> ...] ;

where <attribute-clause> is

<attribute-type> = <attribute>

or

<attribute-group-type > =

( <component-attribute-type $>$ = <attribute>

$[,<$ component-attribute-type $>=$ <attribute> ... ] )

\section{Examples:}

modify entity PAYROLL-SYSTEM with

external-security = "confidential",

identification-names =

(alternate-name = "PAYROLL-SYS",

alternate-name-context = "DIVISION-100");

modify entity AS

entity descriptive-name = ACCOUNTING-SYSTEM; 


\section{Prototype Response:}

Entity <access-name> modified.

\subsubsection{DELETE ENTITY}

\section{Syntax:}

DELETE ENTITY <access-name> [ , access-name ... ] ;

\section{Examples:}

delete entity u8a-30;

delete entity u8a-30, u8a-31, u8a-32;

\section{Prototype Response:}

Entity <access-name> deleted.

Entity <access-name> deleted.

\subsubsection{ADD RELATIONSHIP}

\section{Syntax:}

ADD RELATIONSHIP

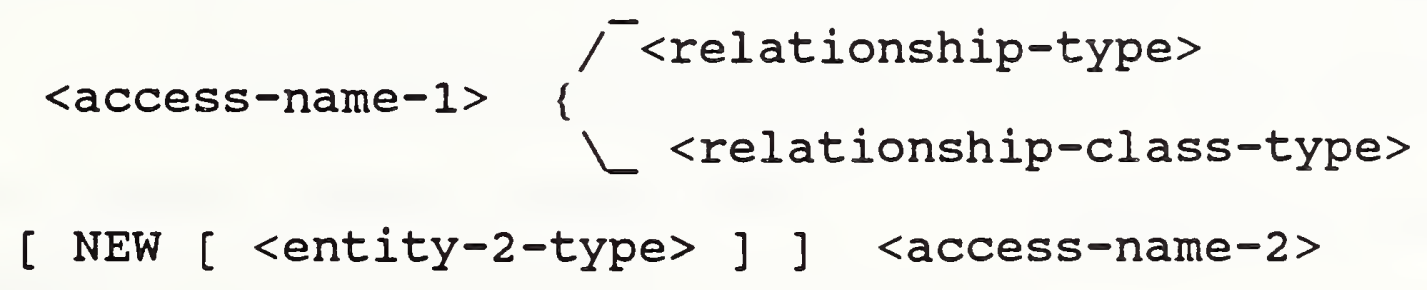

[ WITH [ATTRIBUTES] <attribute-type> = <attribute> $[$ [ <attribute-type $=$ <attribute> ... ] ] ; 


\section{Examples:}

add relationship us system-contains-system $u 8 a_{\text {; }}$

add relationship u8 contains new system u8a-30;

add relationship u8 system-contains-system new u8a-30;

add relationship $u-8$ processes payroll

with access-method = "protected", frequency $=$

"bi-monthly";

\section{Prototype Response:}

Relationship <access-name-1> <relationship-type>

$<$ access-name-2> added.

\subsubsection{MODIFY RELATIONSHIP}

\section{Syntax:}

MODIFY RELATIONSHIP

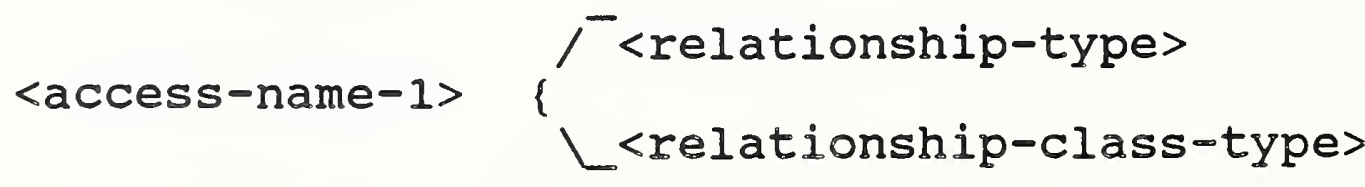

[ WITH [ATTRIBUTES] <attribute-type> = <attribute> [, <attribute-type $\rangle=\langle$ attribute...$]$ ] :

Example:

modify relationship u8 processes payroll with frequency $=$ "50", access-method = "direct" ;

\section{Prototype Response:}

Relationship <access-name-1> <relationship-type> $<$ access-name-2> modified. 


\subsubsection{DELETE RELATIONSHIP}

\section{Syntax:}

DELETE RELATIONSHIP

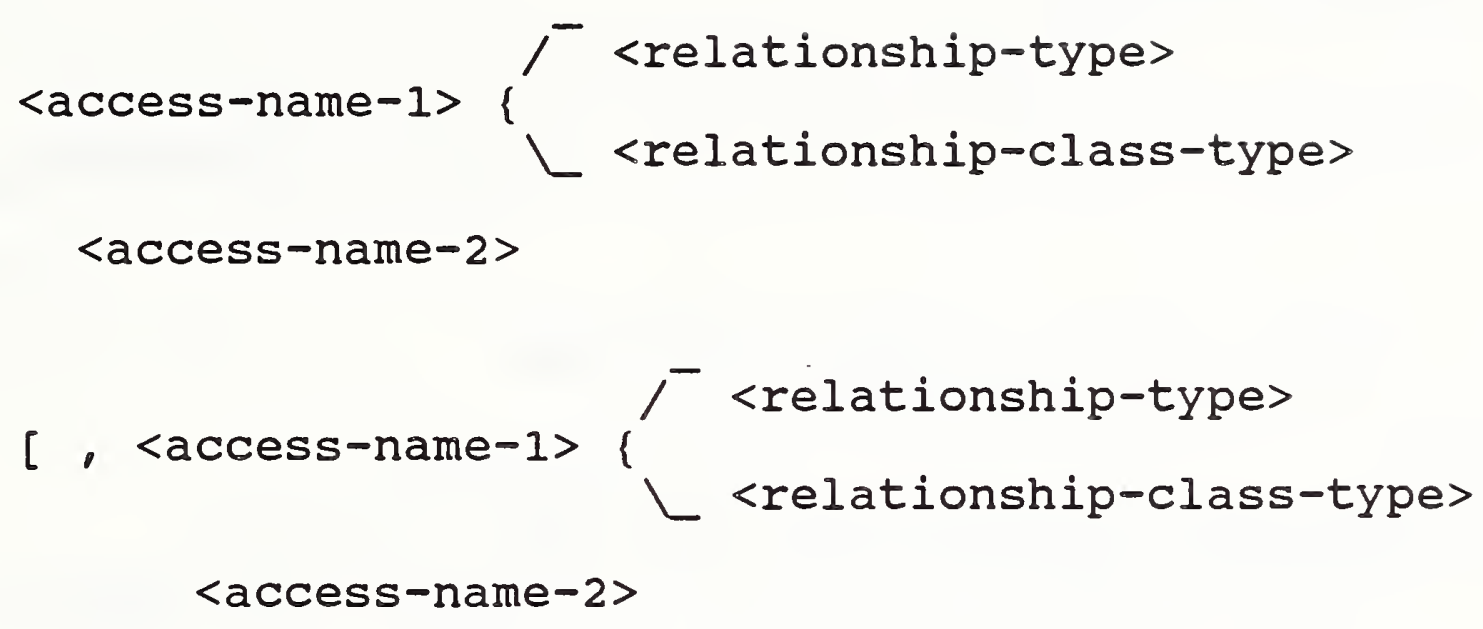

$$
\text { ... ] ; }
$$

\section{Examples:}

delete relationship u8 system-contains-system u8-30; delete relationship us contains u8-25, u8 contains u8a;

\section{Prototype Response:}

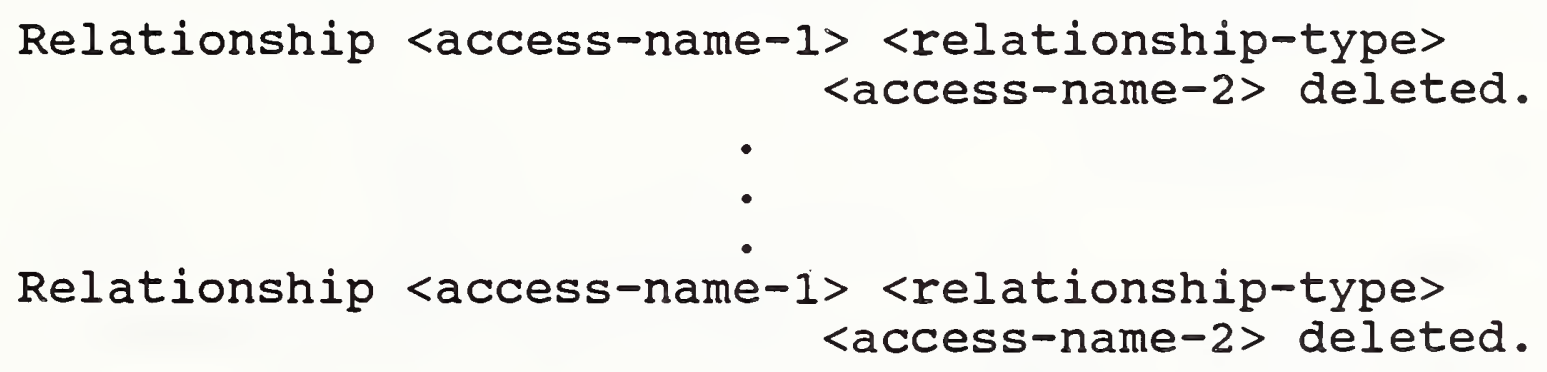

Relationship <access-name-1> <relationship-type> $<$ access-name-2> deleted. (1)

Relationship <access-name-1> <relationship-type> <access-name-2> deleted.

\subsubsection{MODIFY ENTITY ACCESS-NAME}

\section{Syntax:}

MODIFY ENTITY ACCESS-NAME FROM <old-name> TO <new-name> ; 
Example:

modify entity access-name from u8-20 to test1;

Prototype Response:

Entity access-name modified from <old-name> to $<$ new-name $>$.

\subsubsection{MODIFY ENTITY DESCRIPTIVE-NAME}

\section{Syntax:}

MODIFY ENTITY DESCRIPTIVE-NAME FROM <old-name>

To <new-name> :

Example:

modify entity descriptive-name from

old-Long-Name-1234567890 to New-Long-Name-1234567890:

\section{Prototype Response:}

Entity descriptive-name modified from <old-name> to

<new-name> for <access-name>.

\section{2 .9 COPY ENTITY}

\section{Syntax:}

COPY ENTITY <access-name-1> [ WITH RELATIONSHIPS ]

TO <access-name-2>

[ ENTITY DESCRIPTIVE-NAME = <descriptive-name> ] ;

\section{Examples:}

copy entity u8-20-10 with relationships to New-u8-20-10;

copy entity Tape recording to Memoirs

entity descriptive-name = Life_and_Times; 


\section{Prototype Response:}

Entity <access-name-1> copied to

$$
\text { entity <access-name-2>. }
$$

\section{2 .10 GENERAL OUTPUT}

\section{Syntax:}

OUTPUT IRD

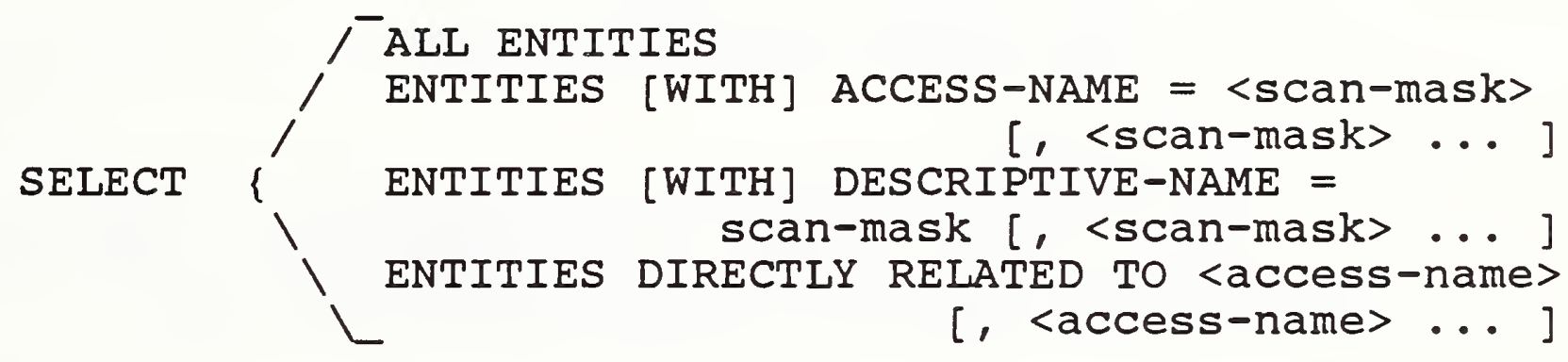

[WHERE

< conditional expression using "(", ")", "AND", "OR" and subexpressions:

ENTITY-TYPE = <entity-type> [, <entity-type>...] ENTITY ASSIGNED ACCESS-NAME <rel-op>

ENTITY ASSIGNED DESCRIPTIVE-NAME <rel-op>

$<$ access-name> $<$ descriptive-name $>$ <attribute-type> <rel-op> <attribute> > ]

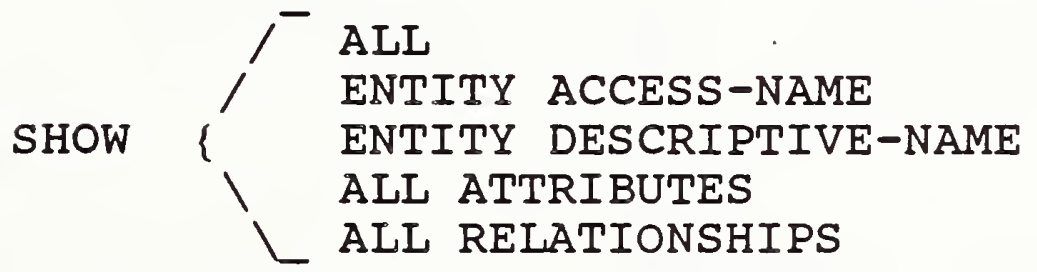

;

A <scan-mask> may use, in addition to explicitly specified characters, the substitution characters "*" and "?". Substitution character "*" matches any sequence of characters, including the null sequence; substitution character "?" matches any single character other than null. The term <rel-op> refers to one of the operators: 


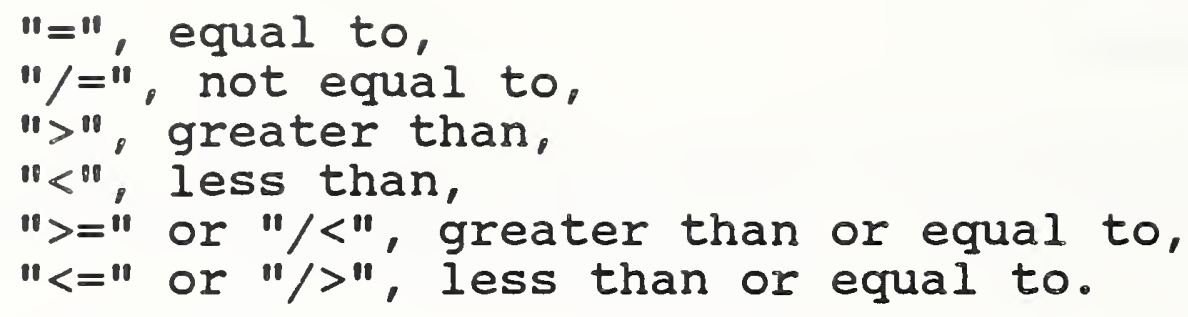

\section{Examples:}

output ird select all show all;

output ird

select entities with access-name $=*$ database*, dbms* where entity-type = file

show entity access-name;

\section{Prototype Response:}

For each of the entities in a hypothetical IRD reported on by OUTPUT IRD SELECT ALL SHOW ALL; the Prototype would generate a display looking something like:

$$
\begin{aligned}
& \text { Entity }=\mathrm{H}-\mathrm{I} \\
& \text { Descriptive-Name = Health-Insurance } \\
& \text { Entity-Type = SYSTEM }
\end{aligned}
$$

Attributes

$$
\text { Added-By = Goldfine }
$$

Last-Modified-By $=$ Kirk

$$
\circ
$$

System-Category $=$ Personnel

\section{Attribute-Groups}

Date-Time-Added

System-Date $=19860720$

System-Time $=152654$

Date-Time-Last-Modified

System-Date $=19860723$

system-Time $=093150$ 


\section{Relationships}

H-I SYSTEM-PROCESSES-FILE H-I-Carrier ACCESS-METHOD = Direct FREQUENCY = Weekly

J_Smith USER-RUNS-SYSTEM H-I

FREQUENCY $=$ Daily

0

0

$\circ$

At the end of the output, the following message is displayed:

IRD output completed.

\subsection{IRD-SCHEMA COMMANDS}

\subsubsection{ADD META-ENTITY}

\section{Syntax:}

ADD META-ENTITY <meta-entity-name> META-ENTITY-TYPE =

[ WITH [META-ATTRIBUTES] <meta-entity-type>

$$
\text { <meta-attribute-type> = <meta-attribute> }
$$

$[,<$ meta-attribute-type $>=\langle$ meta-attribute $>.$. ] ] ;

\section{Examples:}

add meta-entity COLOR meta-entity-type = attribute-type;

add meta-entity colOR meta-entity-type = attribute-type with purpose $=$ "this is attribute-type is used to define the color of a DOCUMENT";

\section{Prototype Response:}

Meta-entity <meta-entity-name> added. 


\subsubsection{MODIFY META-ENTITY}

\section{Syntax:}

MODIFY META-ENTITY <meta-entity-name>

WITH [META-ATTRIBUTES] <attribute-type $=$ = <attribute>

$$
[,<a t t r i b u t e-t y p e\rangle=<\text { attribute }>. .] \text { : }
$$

Examples:

modify meta-entity PUBLICATION

with purpose $=$ "this entity-type refers only to formally published documents" :

modify meta-entity COLOR

with maximum-number-of-occurrences $=7$,

format $=$ string :

Prototype Response:

Meta-entity <meta-entity-name> modified.

\subsubsection{DELETE META-ENTITY}

\section{Syntax:}

DELETE META-ENTITY <meta-entity-name>

[WITH META-RELATIONSHIPS] ;

Example:

delete meta-entity u8a-30;

Prototype Response:

Meta-entity <meta-entity-name> deleted. 


\subsubsection{ADD META-RELATIONSHIP}

\section{Syntax:}

ADD META-RELATIONSHIP

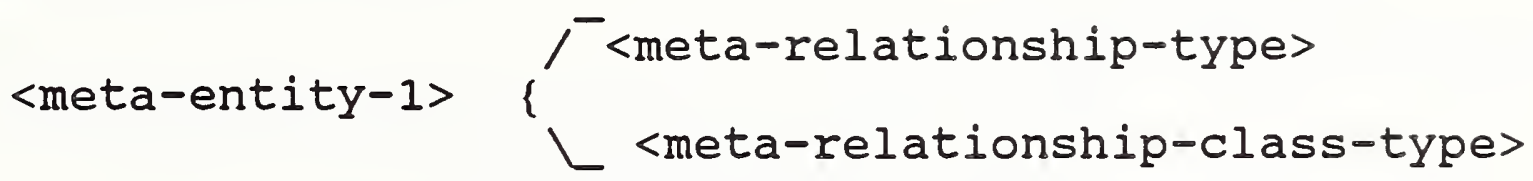

$$
\begin{aligned}
& <\text { meta-entity-2> [ POSITION }=\langle n\rangle] \\
& \text { [WITH [META-ATTRIBUTES] } \\
& \text { <meta-attribute-type> = <meta-attribute> } \\
& {[,<\text { meta-attribute-type }>=<\text { meta-attribute> ... ] ] ; }}
\end{aligned}
$$

\section{Example:}

add meta-relationship

document-contains-program connects document position $=1$ with purpose $=$ "example";

\section{Prototype Response:}

Meta-relationship <meta-entity-1>

<meta-relationship-type> <meta-entity-2> added.

\subsubsection{MODIFY META-RELATIONSHIP}

\section{Syntax:}

MODIFY META-RELATIONSHIP

$$
\begin{aligned}
& \text { <meta-entity-1> }\left\{\begin{array}{l}
/ \text { <meta-relationship-type> } \\
\backslash \text { <meta-relationship-class-type> }
\end{array}\right. \\
& <\text { meta-entity-2> [ POSITION }=\langle\mathrm{n}\rangle] \\
& \text { [WITH [META-ATTRIBUTES] } \\
& <\text { meta-attribute-type }>\text { = <meta-attribute> } \\
& {[,<\text { meta-attribute-type }>=\text { <meta-attribute> ... ] ] ; }}
\end{aligned}
$$




\section{Example:}

modify meta-relationship

document-contains-program connects program position $=2$ with purpose = "another example";

\section{Prototype Response:}

Meta-relationship <meta-entity-1> <meta-relationship-type> <meta-entity-2> modified.

\subsubsection{DELETE META-RELATIONSHIP}

\section{Syntax:}

DELETE META-RELATIONSHIP

$$
\begin{aligned}
& \text { / <meta-relationship-type> } \\
& \text { <meta-entity-1> }\{\backslash \text { <meta-relationship-class-type> } \\
& \text { <meta-entity-2> [ POSITION }=\langle\mathrm{n}\rangle] \text {; }
\end{aligned}
$$

Example:

delete meta-relationship

document-contains-program connects program

position $=2$ i

\section{Prototype Response:}

Meta-relationship <meta-entity-1>

<meta-relationship-type> <meta-entity-2> deleted.

\subsubsection{MODIFY META-ENTITY ACCESS-NAME}

\section{Syntax:}

MODIFY META-ENTITY ACCESS-NAME

FROM <meta-entity-access-name-1>

TO <meta-entity-access-name-2> ; 


\section{Example:}

modify meta-entity access-name from document to report;

\section{Prototype Response:}

$$
\begin{aligned}
& \text { Meta-entity access-name modified from } \\
& <\text { meta-entity-access-name-1> } \\
& \text { to }<\text { meta-entity-access-name-2>. }
\end{aligned}
$$

\section{3 .8 OUTPUT IRD-SCHEMA}

\section{Syntax:}

OUTPUT IRD-SCHEMA

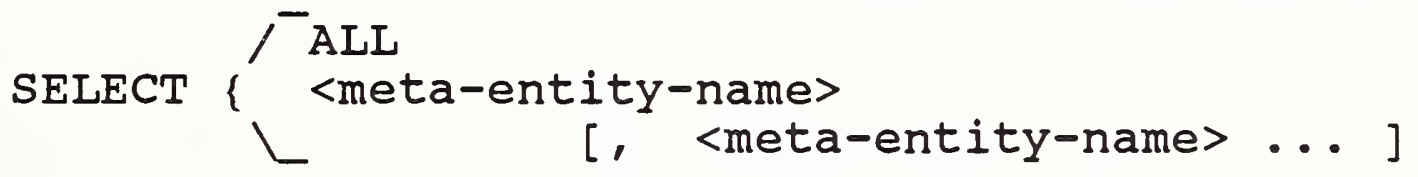

TALL

/ ALL META-ATTRIBUTES

SHOW $\{$ META-ATTRIBUTES

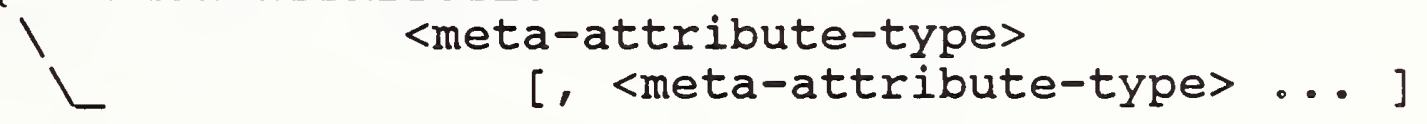

;

Example:

output ird-schema select document show all;

\section{Prototype Response:}

For this command, the Prototype would generate a display looking something like:

Meta-Entity $=$ DOCUMENT

Meta-Entity-Type $=$ ENTITY-TYPE 


\section{Meta-Attributes}

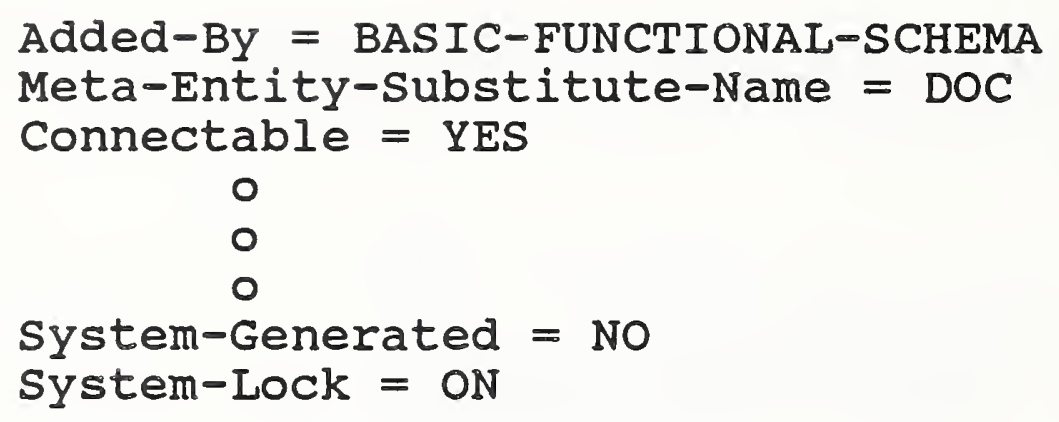

\section{Meta-Relationships}

DOCUMENT ENTITY-TYPE-CONTAINS-ATTRIBUTE-TYPE

Implementation-Lock $=$ OFF

ADDED-BY

○

$\circ$

0

System-Lock $=\mathrm{ON}$

DOCUMENT ENTITY-TYPE-CONTAINS-ATTRIBUTE-TYPE

CLASSIFICATION

Implementation-Lock $=$ OFF

$\circ$

0

0

System-Lock $=$ OFF

$\circ$

$\circ$

0

DOCUMENT ENTITY-TYPE-CONTAINS-ATTRIBUTE-GROUP-TYPE

Implementation-Lock $=$ OFF IDENTIFICATION-NAMES

o

$\circ$

$\circ$ 


\section{System-Lock $=$ OFF}

DOCUMENT-CONTAINS-DOCUMENT

RELATIONSHIP-TYPE-CONNECTS-ENTITY-TYPE

Implementation-Lock $=$ OFF

DOCUMENT

o

0

0

System-Lock $=$ OFF

o

0

0

USER-RESPONSIBLE-FOR-DOCUMENT

RELATIONSHIP-TYPE-CONNECTS-ENTITY-TYPE

Implementation-Lock $=$ OFF

DOCUMENT

o

0

0

System-Lock $=$ OFF

At the end of the output, the following message is displayed:

IRD-SCHEMA output completed.

NOTE: Care should be taken in issuing the command:

output ird-schema select all show all;

This command will cause the display of the entire IRDSchema, which will include the Minimal schema and, unless it has been redefined, the Basic Functional Schema. Over 350,000 characters of text are generated in the display of the Minimal and Basic Functional Schemas.

\subsection{UTILITY COMMANDS}

\subsubsection{CREATE IRD}

\section{Syntax:}

CREATE IRD <IRD-name> IRD-SCHEMA IS STANDARD ; 


\section{Example:}

create ird production-2 ird-schema is standard:

The term "standard" in the Prototype's CREATE IRD command refers to a combination of the Minimal Schema and the Basic Functional Schema of the IRDS standard.

Prototype Response:

INFORMATION IXXXX: Creating 1st schema table

INFORMATION IXXXX: Creating 2nd schema table

INFORMATION IXXXX: Creating 3rd schema table

INFORMATION IXXXX: Creating lst data table

INFORMATION IXXXX: creating 2nd data table

INFORMATION IXXXX: Creating 3rd data table

INFORMATION IXXXX: All done.

\section{4 .2 REMOVE IRD}

\section{Syntax:}

REMOVE IRD <IRD-name> ;

Example:

remove ird test-04;

Prototype Response:

IRD <IRD-name> removed.

The Specifications for the IRDS Command Language do not contain a REMOVE IRD command. However, the ability to create new IRDs certainly implies the need to remove them. Hence the Prototype was implemented with this command. 


\section{4 .3 EXIT}

\section{Syntax:}

EXIT ;

Example:

exit;

\section{Prototype Response:}

Return to calling program or operating system.

\section{4 .4 HELP}

\section{Syntax:}

HELP [ <command> ] ;

Examples:

help;

help add meta-relationship;

\section{Prototype Response:}

For HELP;, a list of the currently available commands.

For HELP <command>i, a description of the syntax of that command, and some examples of command usage.

\subsection{ERROR MESSAGES}

The Prototype generates all the appropriate error messages specified in the IRDS standard. In addition, certain error conditions that are not documented in the specifications are recognized by the Prototype. These conditions cause the generation of self explanatory error messages beginning with "EXXXXX:" 


\subsection{COMMAND IANGUAGE ABBREVIATIONS}

The Prototype accepts abbreviations for a set of IRDSwords that are defined in the standard and that are part of the Command Language. An abbreviation can be used anywhere in place of its corresponding full formulation.

IRDS-Word

ACCESS-NAME

ALTERNATE-NAME

ASSIGNED

ATTRIBUTES

ATTRIBUTE-TYPE

COPY

CREATE

DELETE

DESCRIPTIVE-NAME

ENTITY-TYPE

META-ATTRIBUTES

META-ENTITY

META-ENTITY-TYPE

META-RELATIONSHIP

META-RELATIONSHIPS

MODIFY

OUTPUT

RELATIONSHIP

RELATIONSHIPS

RELATIONSHIP-TYPE
Abbreviation

NAME

ANAME

ASSGN

ATTRB

ATYPE

CPY

CRE

DEL

DNAME

ETYPE

MATRBS

MENTY

METYPE

MREL

MRELS

MOD

OUT

REL

RELS

RTYPE

The prototype also accepts the set of meta-entity substitute-names, such as DOC for DOCUMENT and SYS-CON-SYS for SYSTEM-CONTAINS-SYSTEM, defined as part of the "standard" schema. Appendices A and B of the IRDS Technical Overview [2] contain a complete list of these substitute-names. 


\section{THE IRDS PROTOTYPE SCHEMA}

\subsection{THE STRUCTURE OF THE SQL TABLES}

Each IRD has associated with it eleven SQL tables, which contain all the IRD and IRD-schema data for that dictionary. These tables are:

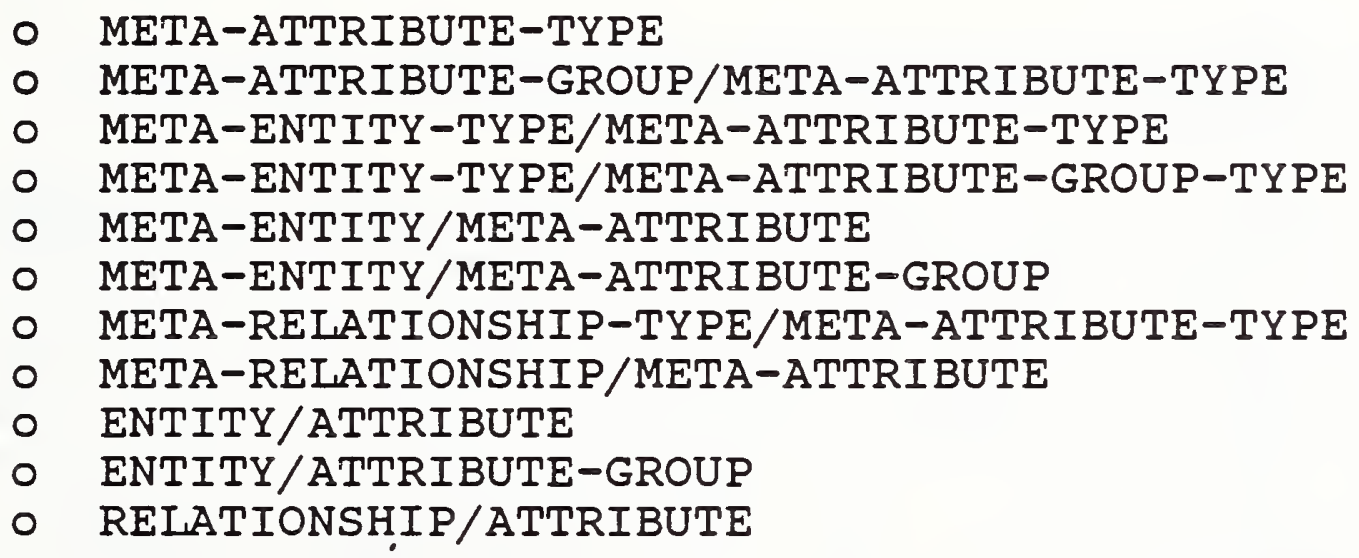

The following sections present the SQL definitions for each of these tables.

\subsubsection{The META-ATTRIBUTE-TYPE Table}

The META-ATTRIBUTE-TYPE table (MATYPE) stores the descriptive information defining the Prototype's metaattribute-types, as specified in section 9.3 of Module 1 of the IRDS. Specifications [1]. Each row of the table corresponds to a meta-attribute-type; the columns could be said to correspond to meta-meta-attribute-types. once the prototype source code is compiled, MATYPE is fixed, in that there is no provision in the standard for a user to be able to redefine meta-attribute-types. Since it is fixed, MATYPE is stored once, and is shared by all IRDs using the given executable.

\section{Definition:}

create table MATYPE

(meta_attribute_type_name inter̃nal name description

format minimum_length

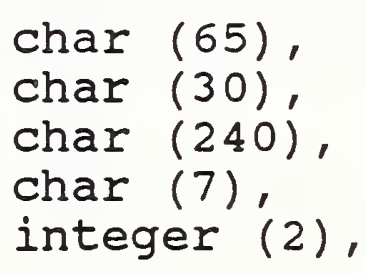




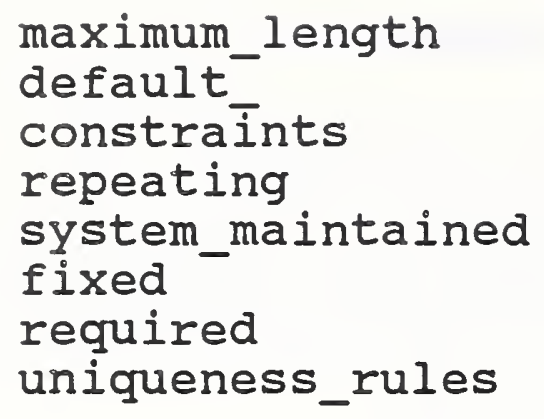

integer (5),
char (20)',
char (240),
char (3),
char (3),
char (3),
char (3)',
char (3)',

\subsubsection{The META-ATTRIBUTE-GROUP-TYPE/META-ATTRIBUTE- TYPE Table}

The META-ATTRIBUTE-GROUP-TYPE/META-ATTRIBUTE-TYPE (MAGTYPE_MATYPE) table describes the association between the meta-attribute-group-types and their component metaattribute-types in the Prototype's IRD-schema, as specified in section 9.6 and Table 3 of Module 1 of the IRDS Specifications. Each row of the table corresponds to a component meta-attribute-type of a meta-attribute-group-type; each column corresponds to a meta-attribute-type. MAGTYPE_MATYPE is fixed, stored once, and shared by all IRDs.

\section{Definition:}

create table MAGTYPE_MATYPE

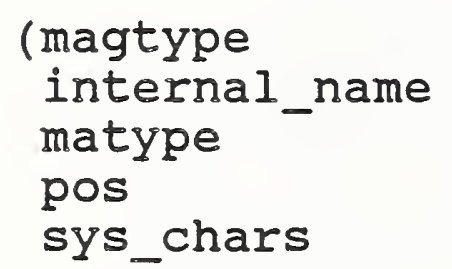

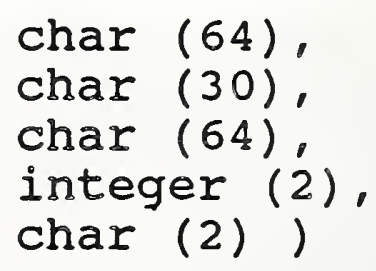

\subsubsection{The META-ENTITY-TYPE/META-ATTRIBUTE-TYPE Table}

The META-ENTITY-TYPE/META-ATTRIBUTE-TYPE (METYPE_MATYPE) table describes the correspondence between the meta-entitytypes and their associated meta-attribute-types in the Prototype's IRD-schema, as specified in section 9.4 and Table 1 of Module 1 of the IRDS Specifications. Each row of the table corresponds to a meta-entity-type; each column corresponds to a meta-attribute-type. METYPE_MATYPE is fixed, stored once, and shared by all IRDs. 


\section{Definition:}

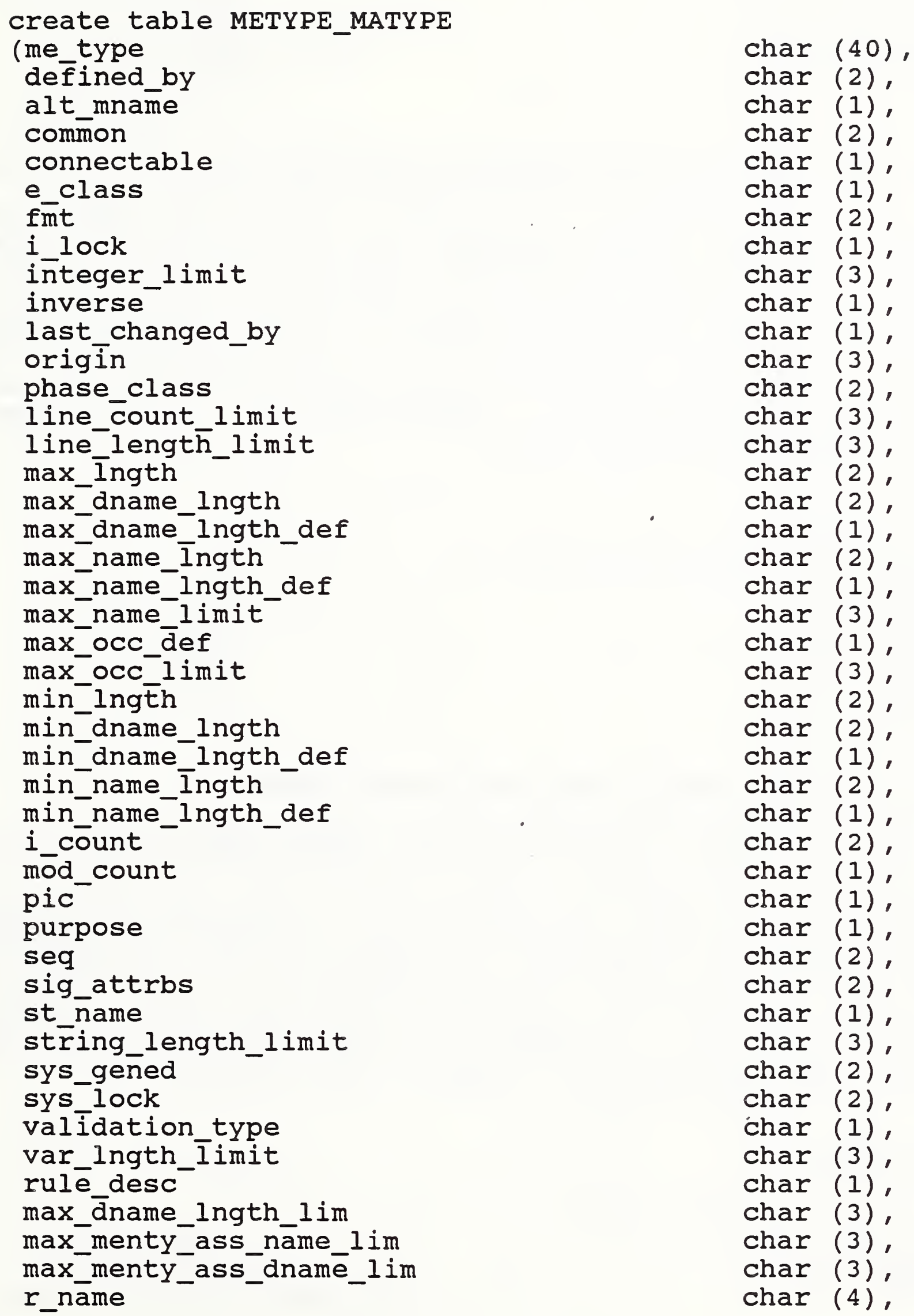




$$
\begin{aligned}
& \text { r_mname } \\
& \text { mode_- } \\
& \text { sys_maint } \\
& \text { grp_txt_alwd } \\
& \text { var }
\end{aligned}
$$
char (4),
char (1),
char (1),
char (3),
char (1) )

\subsubsection{The META-ENTITY-TYPE/META-ATTRIBUTE-GROUP-TYPE Table}

The META-ENTITY-TYPE/META-ATTRIBUTE-GROUP-TYPE (METYPE MAGTYPE) table describes the correspondence between the meta-entity-types and their associated meta-attributegroup-types in the prototype's IRD-schema, as specified by section 9.7 and Table 4 of Module 1 of the IRDS specifications. Each row corresponds to a meta-entity-type; each column corresponds to a component meta attribute-type of a meta-attribute-group-type. METYPE-MAGTYPE is fixed, stored once, and shared by all IRDs.

Definition:

create table METYPE_MAGTYPE
(metype
data_range
data value
added
modified

char (64),
char (1),
char (1),
char (2),
char (2)',

\subsubsection{The META-ENTITY/META-ATTRIBUTE Table}

The META-ENTITY/META-ATTRIBUTE (MENTY MATT) table stores all meta-attributes associated with all meta-entities in the Prototype's IRD-schema. Each row corresponds to a metaentity; each column corresponds to a meta-attribute-type. When a new IRD is created, the table is initially populated with the meta-entities in the Minimal schema and the Basic Functional Schema, as specified in section 10.2 .1 of Module 1 and section 5.1 of Module 2 of the IRDS specifications. As new meta-entities are added to the IRD-schema, they are entered into this table.

Definition:

create table MENTY_MATT

(me type

menty

char (35),

char (64), 
id_number

internal_name

menty_variation_name

menty_revision_number

menty_ass_dname

defined_by

alt_mname

common

connectable

e_class

fimt

i_lock

integer_limit

inverse

last_changed_by

origin

phase_class

Iine_count limit

line_length_limit

max Ingth

max_dname_lngth

max_dname_lngth_def

max_name_Ingth

max_name_lngth_def

max_name_limit

max_occ_def

max_occ_limit

min_lngth

min_dname_lngth

min_dname_lngth_def

min name Ingth

min_name_lngth_def

i_count

mod_count

pic

purpose

seq

sig_attrbs

st name

string_length_limit

sys_gened

sys_lock

validation_type

var

var_lngth_limit

rule desc

max_dname_lngth_lim

max_menty_ass_name_lim

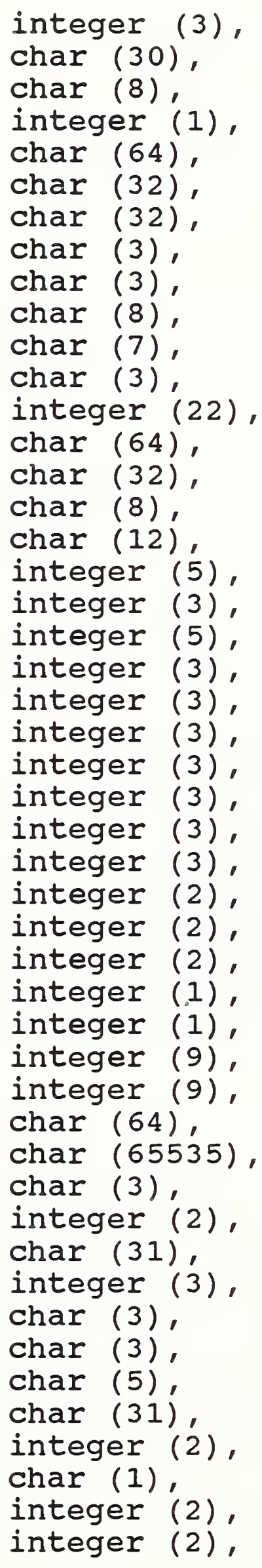




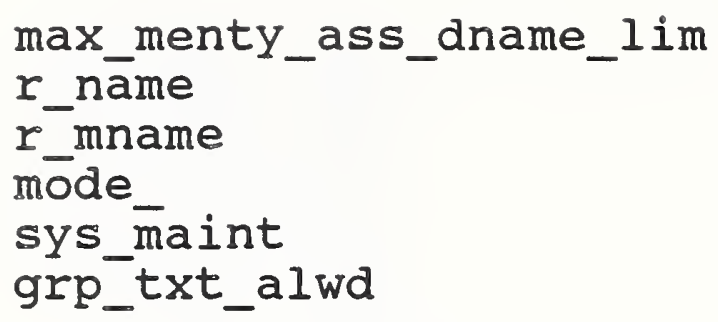

integer (2)
char (1),
char (1),
char (8),
char (3),
char (3)',

\subsubsection{The META-ENTITY/META-ATTRIBUTE-GROUP Table}

The META-ENTITY/META-ATTRIBUTE-GROUP (MENTY MAG) table stores all meta-attribute-groups associated with all metaentities in the Prototype's IRD-schema. Each row corresponds to a meta-entity; each column corresponds to a component meta-attribute-type of a meta-attribute-group-type. When a new IRD is created, the table is initially populated with the meta-entities in the Minimal schema and the Basic Functional Schema, as specified in section 10.2.1 of Module 1 and section 5.1 of Module 2 of the IRDS Specifications. As new meta-entities are added to the IRD-schema, they are entered into this table.

\section{Definition:}

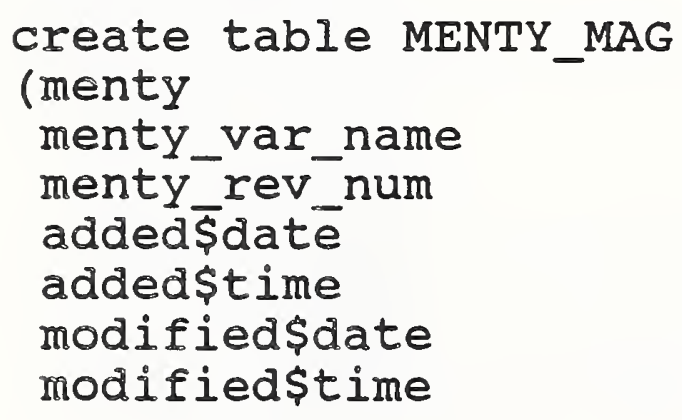

char (64),
char (8),
integer,
char (8),
char (6),
char (8),
char (6)',

\subsubsection{The META-RELATIONSHIP-TYPE/META-ATTRIBUTE-TYPE Table}

The META-RELATIONSHIP-TYPE/META-ATTRIBUTE-TYPE (MRTYPE_MATYPE) table describes the correspondence between the meta-relationship-types and their associated metaattribute-types in the Prototype's IRD-schema, as specified in section 9.5 and Table 2 of Module 1 of the IRDS specifications. Each row corresponds to a meta-relationship-type; each column corresponds to a meta-attribute-type. MRTYPE_MATYPE is fixed, stored once, and shared by all IRDs. 
Definition:

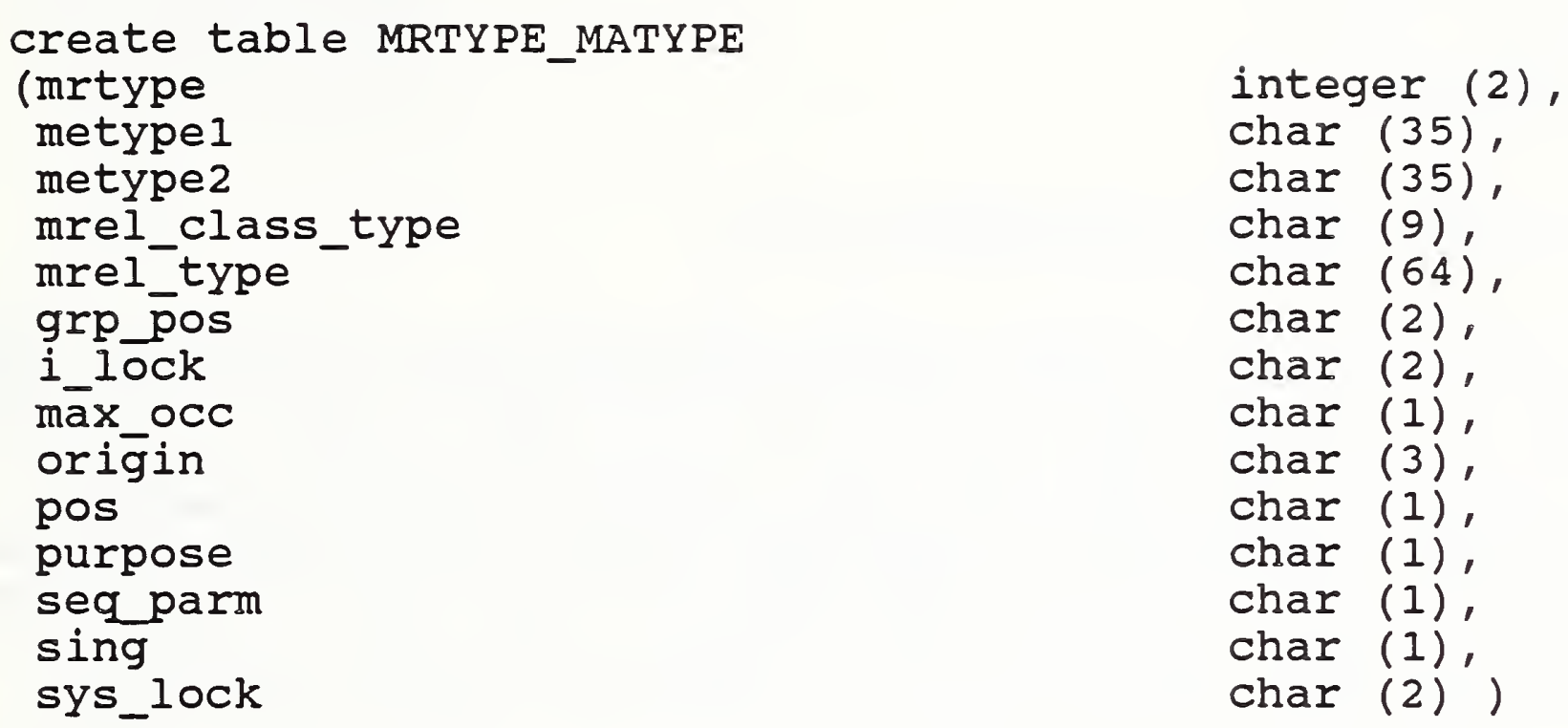

\subsubsection{The META-RELATIONSHIP/META-ATTRIBUTE Table}

The META-RELATIONSHIP/META-ATTRIBUTE (MREL MATT) table stores all meta-attributes associated with all metarelationships in the Prototype's IRD-schema. Each row corresponds to a meta-relationship; each column corresponds to a meta-attribute-type. When a new IRD is created, the table is initially populated with the meta-relationships defined in the Minimal Schema and the Basic Functional Schema, as specified in section 10.3 of Module 1 and section 6 of Module 2 of the IRDS Specifications. As new meta-relationships are added to the IRD-schema, they are entered into this table.

\section{Definition:}
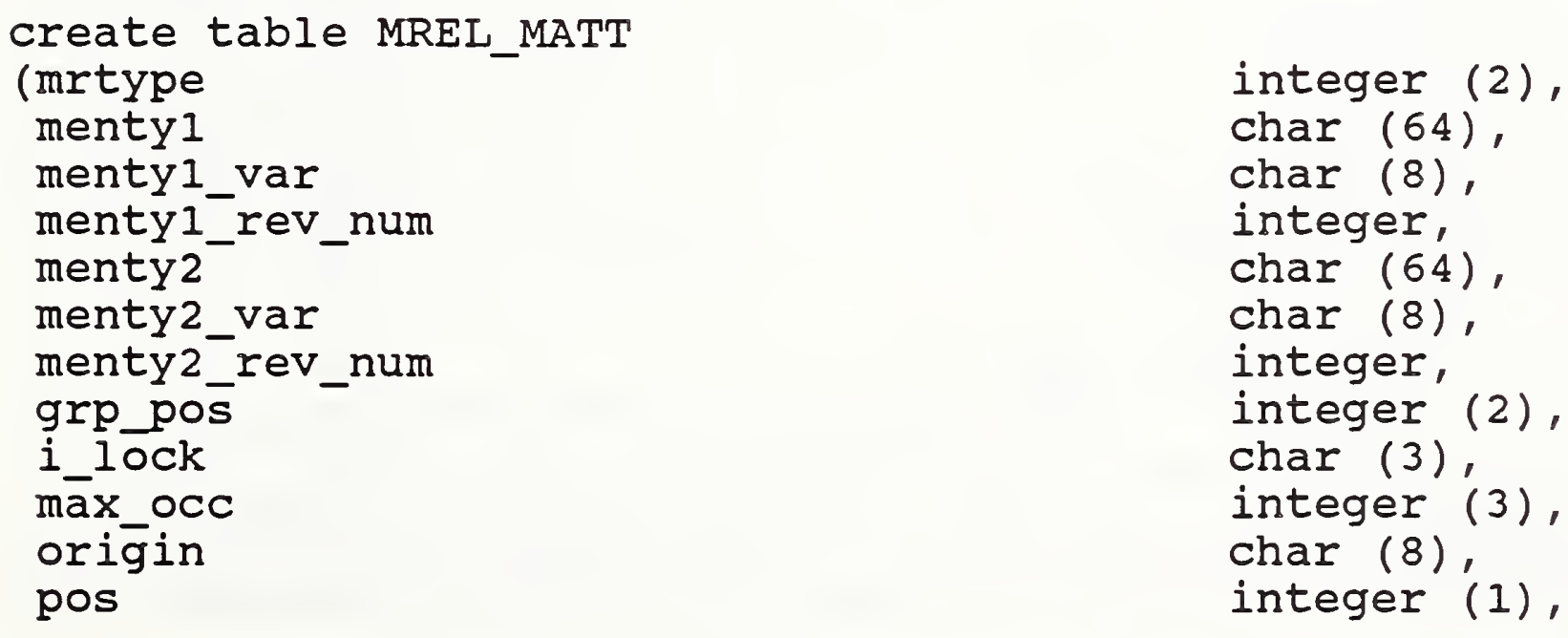


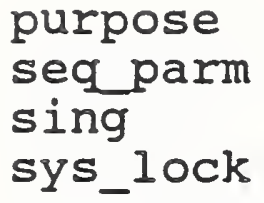

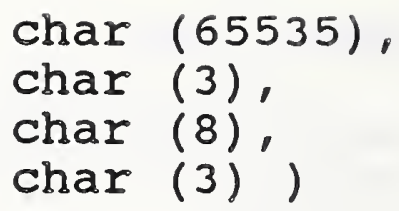

\subsubsection{The ENTITY/ATTRIBUTE Table}

The ENTITY/ATTRIBUTE (ENTY_ATT) table stores all attributes associated with all entities in the application IRD. Each row corresponds to an entity; each column corresponds to an attribute-type defined in the schema of the application IRD. The table is empty when the IRD is created. As entities are added to the IRD, they are entered into this table. When new attribute-types are defined in the schema, corresponding columns are added to the table, making the table dynamic with respect to columns as well as rows.

The following definition is not an extract from the Prototype source code, but is equivalent to that more dynamic definition:

\section{Definition:}

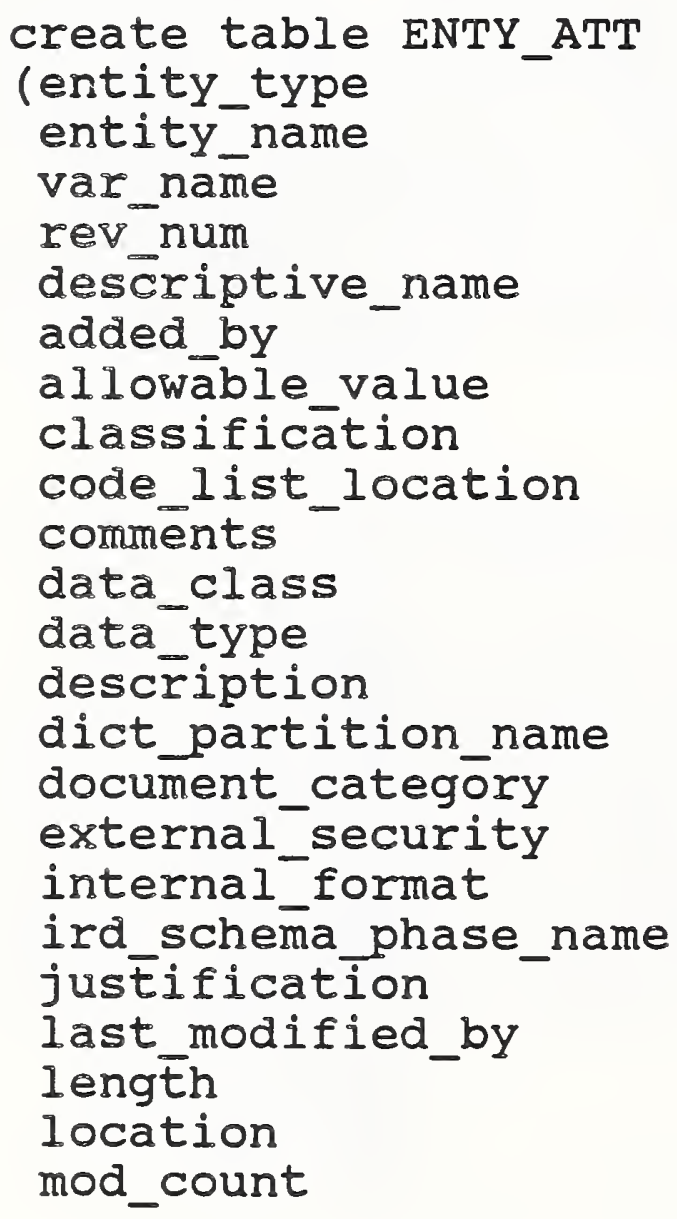

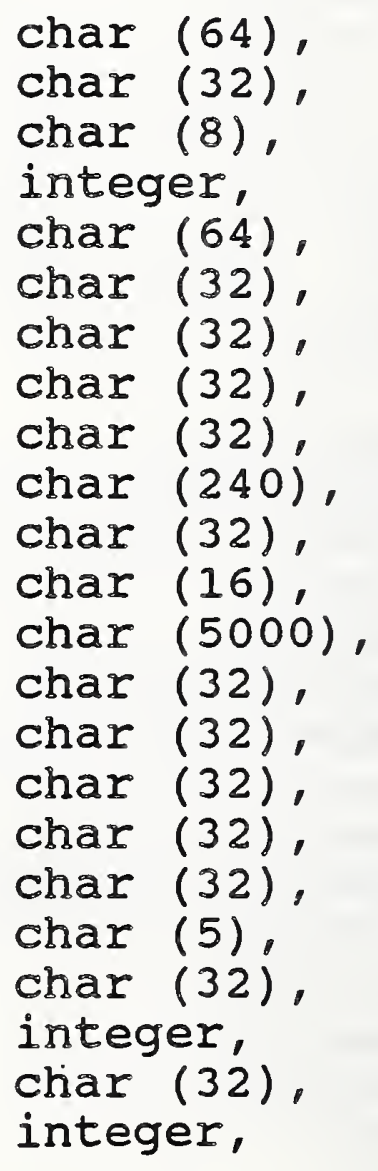


integer_of_records

num_Iiness_code

precision

record_category

scale

system_category

usage integer,

integer,

integer (2),

char (32),

integer (2),

char (32),

char (32) )

\subsubsection{The ENTITY/ATTRIBUTE-GROUP Table}

The ENTITY/ATTRIBUTE-GROUP (ENTY_AG) table stores all attribute-groups associated with all entities in the application IRD. Each row corresponds to an entity; each column corresponds to a component attribute-type of an attributegroup-type defined in the schema of the application IRD. The table is empty when the IRD is created. As entities are added to the IRD, they are entered into this table. When new attribute-group-types are defined in the schema, corresponding columns are added to the table, making the table dynamic with respect to columns as well as rows.

The following definition is equivalent to the definition found in the source code:

\section{Definition:}

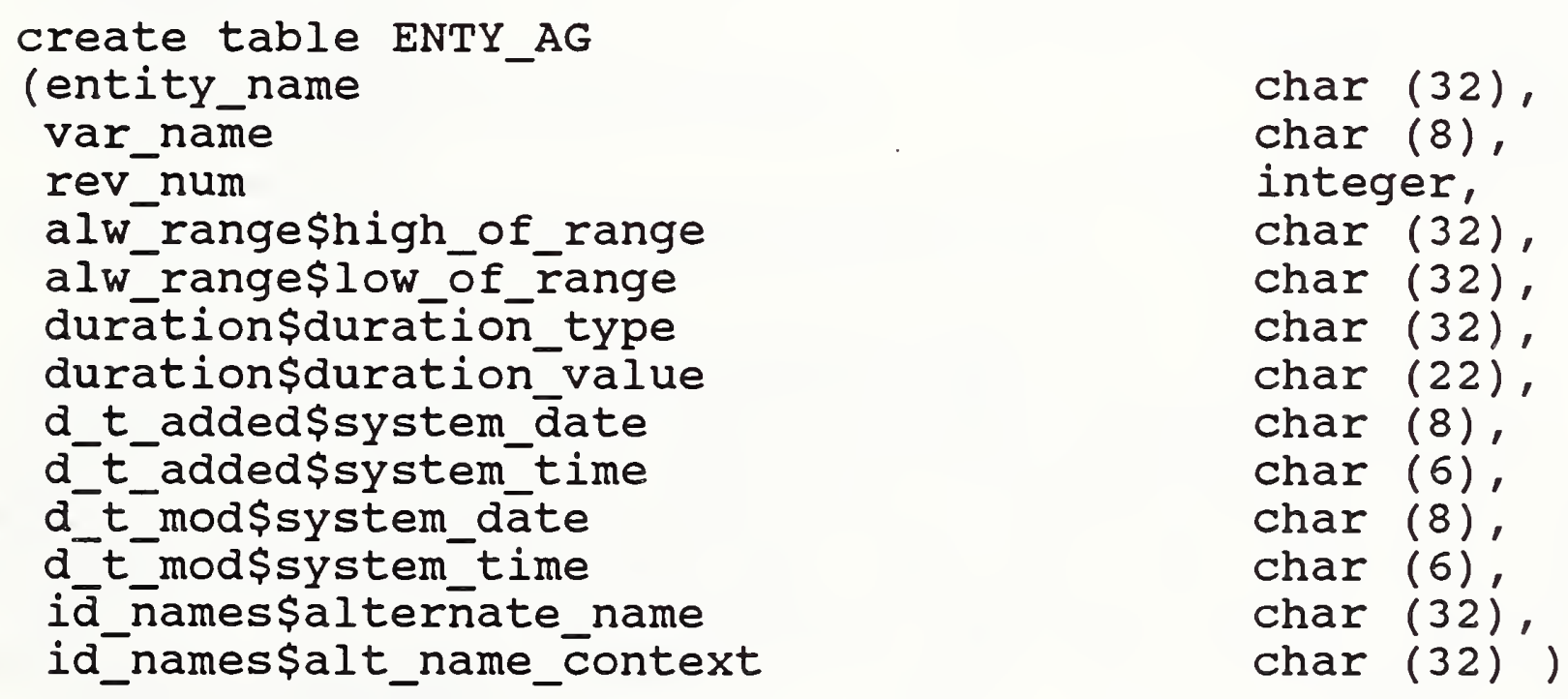

\subsubsection{The RELATIONSHIP/ATTRIBUTE Table}

The RELATIONSHIP/ATTRIBUTE (REL_ATT) table stores all attributes associated with all relationships in the application IRD. Each row corresponds to a relationship; each col- 
umn corresponds to an attribute-type defined in the schema of the application IRD. The table is empty when the IRD is created. As relationships are added to the IRD, they are entered into this table. When new attribute-types are defined in the schema, corresponding columns are added to the table, making the table dynamic with respect to columns as well as rows.

\section{Definition:}

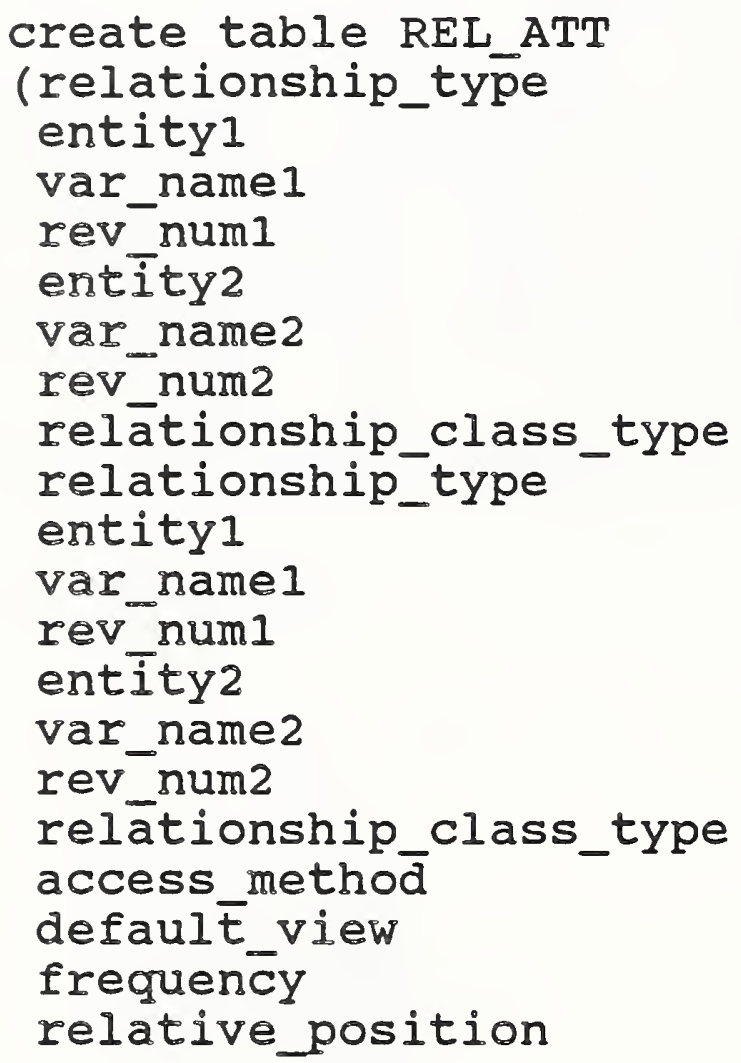

char (64),
char (32),
char (8),
integer,
char (32),
char (8),
integer,
char (64),
char (64),
char (32),
char (8),
integer,
char (2),
char (8),
integer,
char (64),
char (32),
char (3),
char (32),
integer (22) )

\subsection{IMPLEMENTOR DEFINED VALUES IN THE IRDS PROTOTYPE}

The IRDS Standard specifications [1] characterize many of the meta-meta-attributes and meta-attributes in the above tables as "implementor defined" or "installation specified" when applied to specific meta-attribute-types or metaentities. The following sections list the values used in the Prototype for these meta-meta-attributes and metaattributes.

\subsubsection{Values For Meta-Attribute-Types}

ADDED-BY

Maximum Length $=32$ 


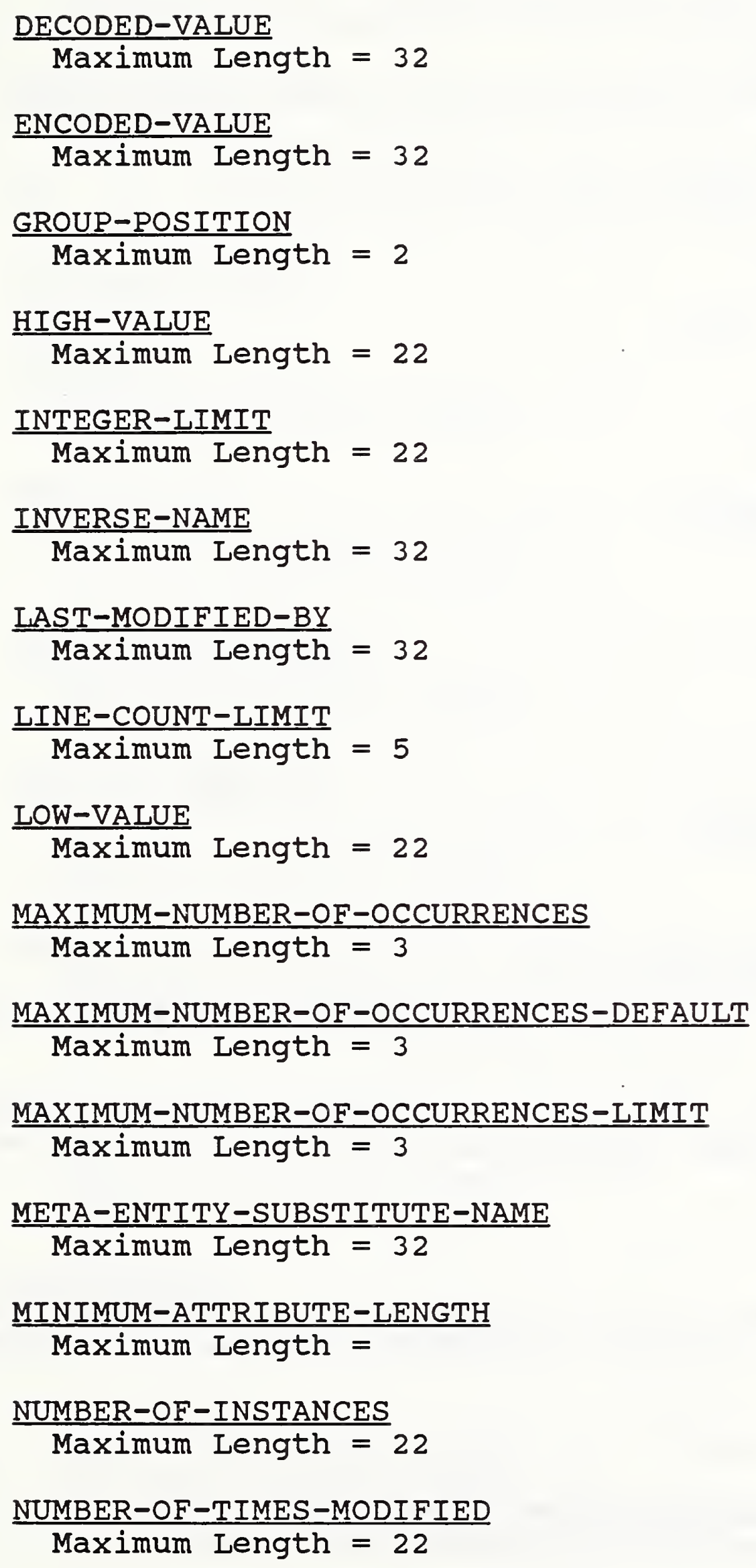




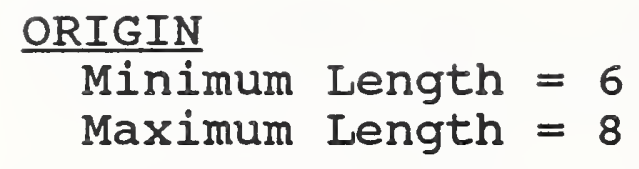

PICTURE

Maximum Length $=32$

PURPOSE

Minimum Length $=1$

Maximum Length $=5000$

SEQUENCE-PARAMETER

Minimum Length $=2$

Maximum Length $=3$

SIGNIFICANT-ATTRIBUTES

Maximum Length $=2$

START-NAME

Maximum Length $=8$

VARIATION

Maximum Length $=2$

VARIATION-LENGTH-LIMIT

Maximum Length $=2$

\subsubsection{Values For Meta-Entities}

The following are the implementor defined metaattributes for the "Standard IRD-Schema" meta-entities:

Each meta-entity has either MINIMAL-SCHEMA or BASICFUNCTIONAL-SCHEMA, as appropriate, as its Added-By metaattribute.

\section{Entity-Types}

Each entity-type has for its Meta-Entity-Substitute-Name the value given in sections $A .1$ and $B .1$ of the IRDS Technical overview [2].

For each entity-type:

Maximum-Entity-Assigned-Access-Name-Length $=32$

Maximum-Entity-Assigned-Descriptive-Name-Length $=64$ 
Minimum-Entity-Assigned-Access-Name-Length $=1$

Minimum-Entity-Assigned-Descriptive-Name-Length $=1$

\section{Relationship-Types and Relationship-Class-Types}

Each relationship-type and relationship-class-type has for its Meta-Entity-Substitute-Name the value given in sections A.2 and B.2 of the IRDS Technical Overview.

\section{Attribute-Types}

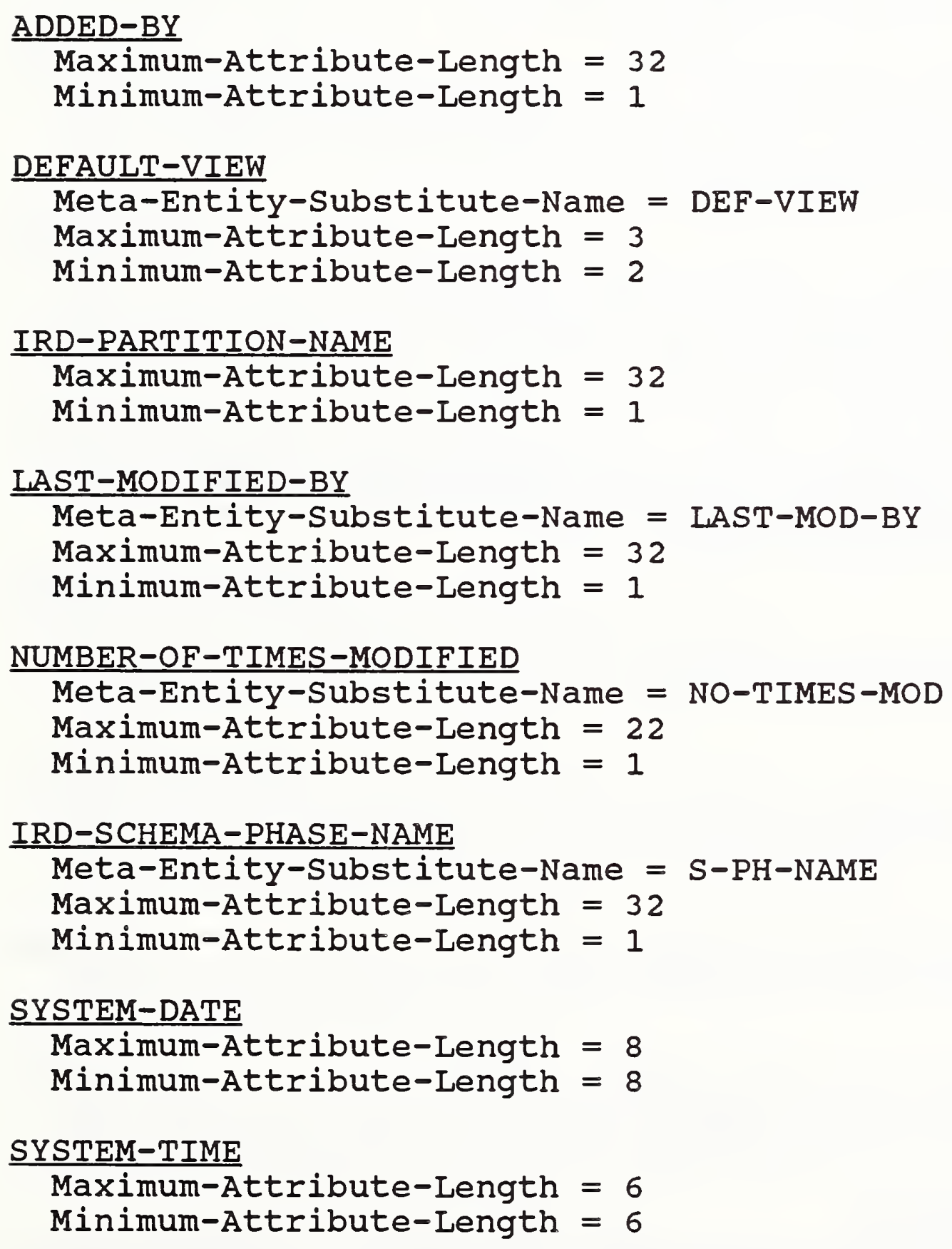




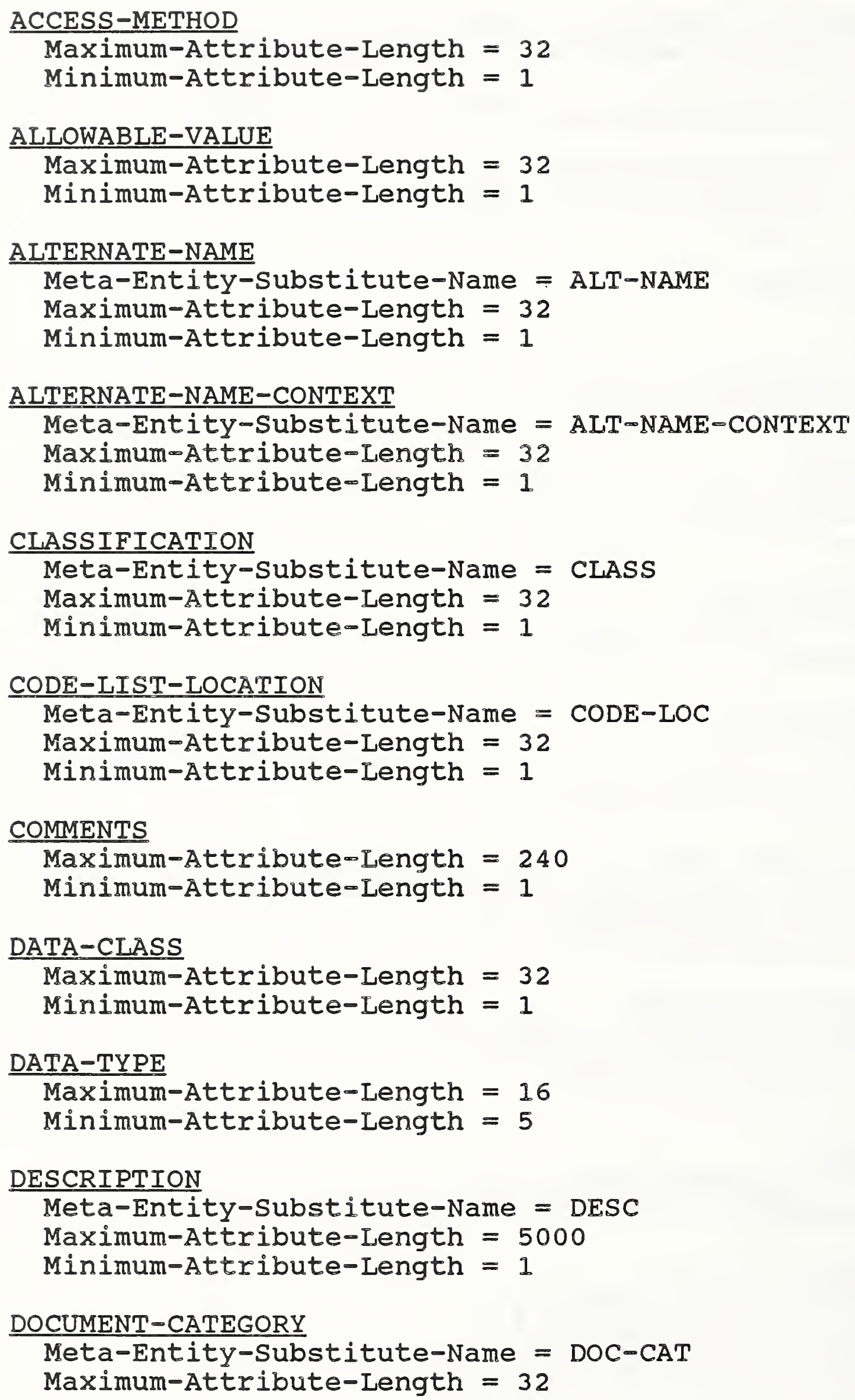




$$
\text { Minimum-Attribute-Length }=1
$$

\section{DURATION-TYPE}

Meta-Entity-Substitute-Name = DUR-TYPE

Maximum-Attribute-Length $=32$

Minimum-Attribute-Length $=1$

\section{DURATION-VALUE}

Meta-Entity-Substitute-Name $=$ DUR-VAL

Maximum-Attribute-Length $=22$

Minimum-Attribute-Length $=1$

\section{EXTERNAL-SECURITY}

Meta-Entity-Substitute-Name = SEC

Maximum-Attribute-Length $=32$

Minimum-Attribute-Length $=1$

\section{FREOUENCY}

Meta-Entity-Substitute-Name $=$ FREQ

Maximum-Attribute-Length $=32$

Minimum-Attribute-Length $=1$

\section{HIGH-OF-RANGE}

Meta-Entity-Substitute-Name $=\mathrm{HIGH}$

Maximum-Attribute-Length $=32$

Minimum-Attribute-Length $=1$

\section{INTERNAL-FORMAT}

Meta-Entity-Substitute-Name $=$ INTF

Maximum-Attribute-Length $=32$

Minimum-Attribute-Length $=1$

\section{JUSTIFICATION}

Meta-Entity-Substitute-Name $=$ JUS

Maximum-Attribute-Length $=5$

Minimum-Attribute-Length $=4$

\section{LENGTH}

Maximum-Attribute-Length $=22$

Minimum-Attribute-Length $=1$

\section{LOCATION}

Meta-Entity-Substitute-Name $=$ LOC

Maximum-Attribute-Length $=32$

Minimum-Attribute-Length $=1$

LOW-OF-RANGE

Meta-Entity-Substitute-Name = LOW 
Maximum-Attribute-Length $=32$

Minimum-Attribute-Length $=1$

NUMBER-OF-LINES-OF-CODE

Meta-Entity-Substitute-Name = NO-LINES-CODE

Maximum-Attribute-Length $=22$

Minimum-Attribute-Length $=1$

NUMBER-OF-RECORDS

Meta-Entity-Substitute-Name = NO-OF-RECS

Maximum-Attribute-Length $=22$

Minimum-Attribute-Length $=1$

PRECISION

Maximum-Attribute-Length $=2$

Minimum-Attribute-Length $=1$

RECORD-CATEGORY

Meta-Entity-Substitute-Name = REC-CAT

Maximum-Attribute-Length $=32$

Minimum-Attribute-Length $=1$

RELATIVE-POSITION

Meta-Entity-Substitute-Name = REL-POS

Maximum-Attribute-Length $=22$

Minimum-Attribute-Length $=1$

SCAIE

Meta-Entity-Substitute-Name = SCL

Maximum-Attribute-Length $=2$

Minimum-Attribute-Length $=1$

SYSTEM-CATEGORY

Meta-Entity-Substitute-Name = SYS-CAT

Maximum-Attribute-Length $=32$

Minimum-Attribute-Length $=1$

USAGE

Maximum-Attribute-Length $=32$

Minimum-Attribute-Length $=1$

\section{IRDS-Defaults}

EXISTING-IRDS-DEFAULTS

Format $=$ STRING

Maximum-Attribute-Length $=32$

Maximum-Entity-Assigned-Descriptive-Name-Length $=64$ 


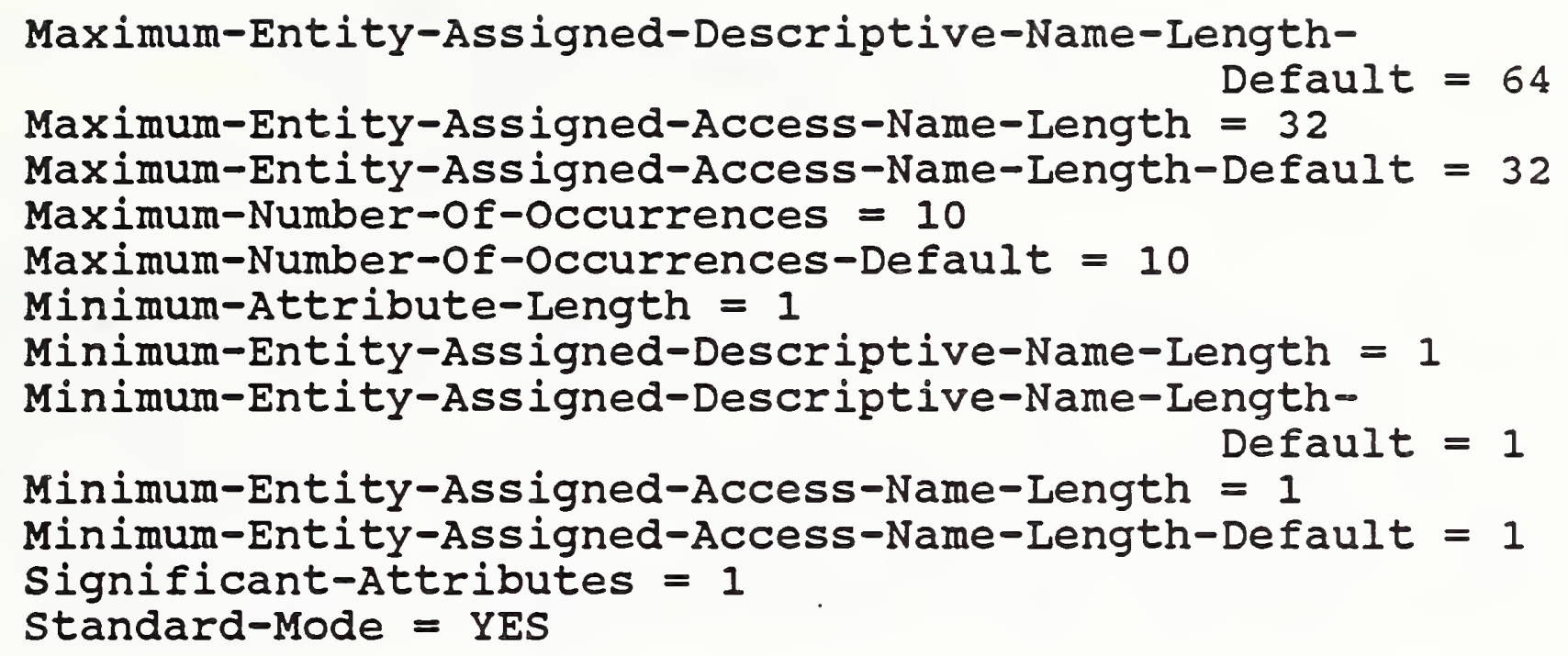

\section{IRDS-Limits}

\section{EXISTING-IRDS-LIMITS}

Integer-Limit $=10000000000000000000000$

Line-Count-Limit $=32767$

Line-Length-Limit $=80$

Maximum-Entity-Assigned-Access-Name-Length-Limit $=32$

Maximum-Entity-Assigned-Descriptive-Name-Length-Limit $=64$

Maximum-Meta-Entity-Assigned-Access-Name-Length-Limit $=32$

Maximum-Meta-Entity-Assigned-Descriptive-Name-Length-

Maximum-Number-of-Occurrences-Limit $=10$

String-Length-Limit $=72$

Variation-Name-Limit $=8$ 


\section{THE IRDS PROTOTYPE SOURCE CODE}

\subsection{OVERVIEW}

The C language Prototype program translates IRDS commands into sQL commands and sends these to the oracle database management system, where the database representing the IRD is maintained. The program performs various consistency checks, some of which include calls to the DBMS to access data. Formatting of the output and some of the entity selection is done at the $\mathrm{C}$ program level. The remainder of the selection is done through the DBMS facilities.

\subsection{DICTIONARY SUBROUTINES}

When the user executes the IRDS prototype, the C program looks for the oracle table DICTIONARY_NAMES to get a list of available IRDs. If no such table is found, the subroutine SET DICT will call MK DICT to create the necessary tables. MK DICT creates and fills DICTIONARY NAMES and those tables that are fixed. MK DICT also creates a set of tables that are modifiable, adding prefix $A$ to the name of each such table. MK DICT then fills the new schema level tables, the data for which comes from the file IRDS.TBL. The IRD level tables are then created using the information contained in the schema level tables. The user is then asked to name the new IRD。

If the DICTIONARY_NAMES table exists but is empty, then the Prototype assumes that the static tables and a set of dictionary tables have already been created and filled. In this case, the user is asked to name the IRD.

If there is data in the DICTIONARY NAMES table, then the list of IRDs is displayed to the user for the user's selection.

When the user executes a CREATE IRD command, the program executes the subroutine CRE_DICT, which finds a prefix to use and then creates a new IRD. The subroutine SET_DICT, which is responsible for making sure that the user is $\bar{p}$ laced in the correct IRD, is executed before the user is given a prompt. 


\subsection{PARSING THE COMMANDS}

Preliminary parsing of each IRDS command is performed by the subroutine GETCOM. GETCOM calls subroutines READCOM and INDEXCOMM. READCOM reads in a command from the standard input. INDEXCOMM takes the string of input from READCOM and divides it into words which are stored in the global array WORD. INDEXCOMM also determines which command was typed in, and records this in the global variable NCOMMAND.

Subroutine DO_COMMAND, called after GETCOM, calls subroutine CK_SYNTAXX. CK_SYNTAX calls subroutine MATCH_TEMPLATE, giving it the template for the specific command and the array of words that INDEXCOMM produces. MATCH_TEMPLATE checks, word-by-word, that the template matches the array of words given. MATCH_TEMPLATE will not do any backtracking, instead counting on having unique choices when there are several options.

MATCH_TEMPLATE assumes that the following characters, when the $\bar{y}$ appear in a template, mean special things:

$[\quad]\{\mid\} \#$,

These special meanings are as follows:

- [ and ] surround a part of the command that is optional.

- The construct $\{a|b| c$ \} matches exactly one of $a$, $b$, or $c$, where $a, b$, and $c$ do not have to be simple.

- 'a ' will match 0 or more a's, where a does not have to be simple. The check for another a is made before the check for what comes after the ' in the template, and this should be considered when writing templates.

- The character \# is followed by a number, 1 through 9 , which is the index to be used into an array of linked lists. The word at this position in the input is added to the linked list which has the given index.

Linked lists are used so that instances of the same type of structure can be stored together into fixed places in the array. For example, a list of attributes specified in an ADD or MODIFY command can all be in one place. These linked lists are dynamic, but because what is stored in them gets translated and stored into non-dynamic structures later, 
there is a limit, about 100, to the number of items that can be in a list.

output commands are not completely parsed by MATCH_TEMPLATE, which counts on subroutine WHERE_s to more thoroüghly parse any WHERE clause. WHERE s makes sure that the attribute-types used do exist, and also does other similar checks. WHERE_S uses backtracking to find the correct parse.

\subsection{COMMAND SUBROUTINES}

After a command has been read in and parsed, the linked list of values from the parse is passed by DO COMMAND to the subroutine for that command. Each command has a corresponding subroutine, and each subroutine has, as its name, an abbreviation of the name of the command. The subroutines do the required consistency checking, and translate the command into a SQL command or a series of SQL commands, which are then executed. Examples of constraints that are checked are: modifying only existing entities, adding only one entity with a given access-name, and adding an attribute for an entity only if the entity's type is meta-related to the attribute's type with an entity-type-contains-attribute-type meta-relationship. Some of the checks involve retrieving information out of the oracle database using SQL commands executed through subroutine calls. Some of the checks and actions are common to several commands, and thus have been written as separate subroutines.

\subsection{OCI SUBROUTINES}

The oracle Call Interface, OCI, subroutines are the subroutines supplied by the DBMS. They all start with an 0 and are described in Oracle's Pro*C User's Guide. These subroutines allow SQL commands to be executed against a database in oracle.

\subsection{HLI SUBROUTINES}

The Prototype's C program contains a special set of subroutines, the name of each member of which starts with HLI. This is an attempt at a consistent interface to the DBMS that both eliminates the repeated writing of certain sequences of calls to oracle's OCI subroutines, and also 
checks for errors. Not all of the calls to the OCI subroutines in the rest of the code have been replaced, but the number has been reduced. This effort has helped to place the direct interface to the DBMS into a limited area of the source code.

\subsection{GLOBAL VARIABLES}

There are a few variables that were made global because of their frequent use in different subroutines. These global variables are defined at the top of each source code file. Two of the variables, CURSOR and IDA, were defined for the oracle subroutines to use. WORD is an array of 100 strings that will hold the input after it has been split up into words. NWORDS is the number of words in the array WORD. PREFIX indicates which IRD a user has activated. NCOMMAND records the type of the current command (e.g., ADD ENTITY or OUTPUT IRD). There are a few global variables that are defined near the definition of a subroutine, and which are used only in that subroutine or set of subroutines.

\subsection{PROGRAM DATA STRUCTURES}

In each of the source code files, types are defined before the global variables are defined. Most of the types defined are structures. There are separate structures that store information about entities, relationships, and attributes, and similar ones that store information at the schema level.

There are a few static variables. The space for these is allocated in the global area, but the variables can be used only where they are defined. The static variables were used to save values between calls to a subroutine, without making the program responsible for the values.

Constants are defined in the file IRDS.CON. and are all in uppercase. One set of constants is used to allow the variable NCOMMAND to be assigned the name of a command instead of an integer or $a$ string. Using an integer directly as the name of a command is confusing, and using a string would require a sequence of ELSE IF statements to determine which command subroutine to call. There is a set of constants to be used to set the length of strings, but these 
Page 46

constants have not been used consistently enough to allow them to be increased without the likelihood of problems arising. 


\section{INSTALLATION INSTRUCTIONS}

The following are needed to install and run the Prototype:

1. A copy of the Oracle Database Management System

2. A "C" Compiler

3. Two $51 / 4$ inch diskettes, supplied by ICST-NBS. These diskettes are written in DOS double-sided doubledensity format, and contain five ASCII text files. The files are:

$$
\begin{aligned}
& \text { irdsa.c ) } \\
& \text { irdsb.c } \\
& \text { irdsc.c - } \\
& \text { irds.con } \\
& \text { irds.tbl }
\end{aligned}
$$

To install the Prototype, the following steps should be performed in the order given:

1. Transfer the files from the diskettes to the host computer.

2. Choose or create an oracle account for the IRDS tables.

3. Change the

\#define ORACLE_UID "irds/irds"

statement in irds.con by replacing "irds/irds" with the oracle userid/password to be used by the IRDS.

4. Change the

\#define TABLEFILE "dral:[kirk.irds.joe]irds.tbl" statement in irds.con by replacing

"dral: [kirk.irds.joe]irds.tbl" 
with the complete name of the file that irds.tbl is stored in.

5. Compile irdsa.c, irdsb.c, and irdsc.c, using any standard "C" compiler. The Prototype uses oracle version 4 or version 5 HLI subroutines, so the HLI libraries must be linked.

6. Run the executable. The first time it is run it will create and fill the tables it needs.

other than in connection with 3 and 4 above, or in conjunction with a deliberate modification of the source code itself, it's probably not advisable to change any of the constants in irds.con. If you do change any of the constants, the source code must be recompiled. A newly compiled version can use the tables created by a previous version.

If you encounter any problems installing or using the Prototype, please contact Tammy Kirkendall at (301)975-3253 or Alan Goldfine at (301)975-3252. 


\section{REFERENCES}

1. ANSI, American National Standard X3.138-1988, Information Resource Dictionary System, American National Standards Institute, New York, 1988.

2. Goldfine, A. H. and Konig, P. A., A Technical Overview of the Information Resource Dictionary system (Second Edition), NBSIR 88-3700, National Bureau of Standards, Gaithersburg, MD, January, 1988 .

3. Goldfine, A. H., Using the Information Resource Dictionary system Command Language (Second Edition), NBSIR 883701, National Bureau of standards, Gaithersburg, MD, January, 1988 .

4. Law, M. H., Guide to Information Resource Dictionary System Applications: General concepts and strateqic systems Planning, NBS Special Publication 500-152, National Bureau of Standards, Gaithersburg, MD, April, 1988.

5. Newton, J. J., Guide on Data Entity Naming Conventions, NBS Special Publication 500-149, National Bureau of Standards, Gaithersburg, MD, October, 1987. 
NBS-114A (REV. 2-80)

\begin{tabular}{|c|c|c|c|}
\hline $\begin{array}{l}\text { U.S. DEPT. OF COMM. } \\
\text { BIBLIOGRAPHIC DATA } \\
\text { SHEET (See in structions) }\end{array}$ & $\begin{array}{l}\text { 1. PUBLICATION OR } \\
\text { REPORT NO. } \\
\text { NBSIR } 88-3830\end{array}$ & 2. Performing Org & $\begin{array}{l}\text { 3. Publication Date } \\
\text { AUGUST } 1988\end{array}$ \\
\hline \multicolumn{4}{|l|}{ 4. TITLE AND SUBTITLE } \\
\hline \multicolumn{4}{|c|}{ The ICST-NBS Information Resource Dictionary System Command Language Prototype } \\
\hline \multicolumn{4}{|l|}{ 5. $A U T H O R(S)$} \\
\hline \multicolumn{4}{|c|}{ Alan Goldfine, Thomasin Kirkendall } \\
\hline \multicolumn{3}{|c|}{ 6. PERFORMING ORGANIIZATION (If joint or other than NBS, see in structlons) } & 7. Contracu Grane No. \\
\hline \multicolumn{2}{|c|}{ NATIONAL BUREAU OF STANDARDS } & & 8 Tronof Renots \\
\hline \multicolumn{2}{|c|}{ U.S. DEPARTMENT OF COMMERCE } & & \&. Type of Report \& Per \\
\hline
\end{tabular}

9. SPONSORING ORGANIZATION NAME AND COMPLETE ADDRESS (Street。City, Stote, ZIP)

10. SUPPLEMENTARY NOTES

Document describes a computer program; SF-185, FIPS Software Summary, is attached.

11. ABST.RACT (A 200-word or less foctual summary of most significant information. If document includes a significant bibliography or literature survey. mention it here)

This publication is a report on the Information Resource Dictionary System (IRDS) Command Language prototype developed by the Institute for Computer Sciences and Technology of the National Bureau of Standards. It discusses the structure, source code, and operating environment of the Prototype, specifies the precise subset of the standard IRDS Command Language implemented, provides instructions for installing the Prototype software, and leads the reader through a typical user session.

12. KEY WORDS (Six to iwelve entries: alphabetical order: capitalize only proper names: and separate key words by semicolons) command language; data dictionary; data dictionary system; Information Resource Dictionary System; IRDS; prototype.

13. AVAILABILITY

XX Unlimited

For Official Distribution. Do Not Release to NTIS

Order From Superintendent of Documents, U.S. Government Printing Office, Washington. D.C. 20402.

XX Order From National Technical Information Service (NTIS), Springfield, VA. 22161
14. NO. OF PRINTED PAGES

$$
55
$$

15. Price

$\$ 13.95$ 